\title{
Multi-terminal transport measurements of MoS2 using a van der Waals heterostructure device platform
}

\section{Citation}

Cui, Xu, Gwan-Hyoung Lee, Young Duck Kim, Ghidewon Arefe, Pinshane Y. Huang, Chul-Ho Lee, Daniel A. Chenet, et al. 2015. "Multi-Terminal Transport Measurements of MoS2 Using a van Der Waals Heterostructure Device Platform." Nature Nanotechnology 10 (6) (April 27): 534-540. doi:10.1038/nnano.2015.70.

\section{Published Version}

doi:10.1038/nnano.2015.70

\section{Permanent link}

http://nrs.harvard.edu/urn-3:HUL.InstRepos:22557383

\section{Terms of Use}

This article was downloaded from Harvard University's DASH repository, and is made available under the terms and conditions applicable to Other Posted Material, as set forth at http:// nrs.harvard.edu/urn-3:HUL.InstRepos:dash.current.terms-of-use\#LAA

\section{Share Your Story}

The Harvard community has made this article openly available.

Please share how this access benefits you. Submit a story.

Accessibility 


\section{Multi-terminal transport measurements of $\mathrm{MoS}_{2}$ using a van der Waals heterostructure device platform}

Xu Cui ${ }^{1 \ddagger}$, Gwan-Hyoung Lee ${ }^{2 \ddagger^{*}}$, Young Duck Kim ${ }^{1 \ddagger}$, Ghidewon Arefe ${ }^{1}$, Pinshane Y. Huang ${ }^{3}$, Chul-Ho Lee ${ }^{4}$, Daniel A. Chenet ${ }^{1}$, Xian Zhang ${ }^{1}$, Lei Wang ${ }^{1}$, Fan Ye ${ }^{5}$, Filippo Pizzocchero ${ }^{6}$, Bjarke S. Jessen ${ }^{6}$, Kenji Watanabe ${ }^{7}$, Takashi Taniguchi ${ }^{7}$, David A. Muller ${ }^{3,8}$, Tony Low ${ }^{9}$, Philip $\mathrm{Kim}^{10}$, and James Hone ${ }^{1 *}$

${ }^{1}$ Department of Mechanical Engineering, Columbia University, New York, NY 10027, USA

${ }^{2}$ Department of Materials Science and Engineering, Yonsei University, Seoul 120-749, Republic of Korea

${ }^{3}$ School of Applied and Engineering Physics, Cornell University, Ithaca, NY 14853, USA

${ }^{4}$ KU-KIST Graduate School of Converging Science and Technology, Korea University, Seoul 136-701, Republic of Korea

${ }^{5}$ Department of Material Science and Engineering, Columbia University, New York, NY 10027, USA

${ }^{6}$ DTU Nanotech, Technical University of Denmark, Ørsteds Plads, 345E, Kgs. Lyngby 2800, Denmark

${ }^{7}$ National Institute for Materials Science, 1-1 Namiki, Tsukuba 305-0044, Japan

${ }^{8}$ Kavli Institute at Cornell for Nanoscale Science, Ithaca, NY 14853, USA

${ }^{9}$ Department of Electrical \& Computer Engineering, University of Minnesota, Minneapolis, MN 55455, USA

${ }^{10}$ Department of Physics, Harvard University, Cambridge, MA 02138, USA

$\$$ These authors contributed equally.

Corresponding authors: gwanlee@yonsei.ac.kr and jh2228@columbia.edu 
Atomically thin two-dimensional semiconductors such as $\mathrm{MoS}_{2}$ hold great promise in electrical, optical, and mechanical devices and display novel physical phenomena. However, the electron mobility of mono- and few-layer $\mathrm{MoS}_{2}$ has so far been substantially below theoretically predicted limits, which has hampered efforts to observe its intrinsic quantum transport behaviours. Potential sources of disorder and scattering include both defects such as sulfur vacancies in the $\mathrm{MoS}_{2}$ itself, and extrinsic sources such as charged impurities and remote optical phonons from oxide dielectrics. To reduce extrinsic scattering, here we developed a van der Waals heterostructure device platform where $\mathrm{MoS}_{2}$ layers are fully encapsulated within hexagonal boron nitride, and electrically contacted in a multi-terminal geometry using gate-tunable graphene electrodes. Magneto-transport measurements show dramatic improvements in performance, including a record-high Hall mobility reaching $34,000 \mathrm{~cm}^{2} / \mathrm{Vs}$ for 6-layer $\mathrm{MoS}_{2}$ at low temperature, confirming that low-temperature performance in previous studies was limited by extrinsic interfacial impurities rather than bulk defects in the $\mathrm{MoS}_{2}$. We also observed Shubnikov-de Haas oscillations for the first time in high-mobility monolayer and few-layer $\mathrm{MoS}_{2}$. Modeling of potential scattering sources and quantum lifetime analysis indicate that a combination of short-ranged and long-ranged interfacial scattering limits low-temperature mobility of $\mathrm{MoS}_{2}$. 
Following the many advances in basic science and applications of graphene, other twodimensional (2D) materials, especially transition metal dichalcogenides (TMDCs), have attracted significant interest for their fascinating electrical, optical, and mechanical properties ${ }^{1-8}$. Among the TMDCs, semiconducting $\mathrm{MoS}_{2}$ has been the mostly widely studied: it shows a thicknessdependent electronic band structure ${ }^{3,5}$, reasonably high carrier mobility ${ }^{1,2,6-9}$, and novel phenomena such as coupled spin-valley physics and the valley Hall effect ${ }^{10-14}$, leading to various applications, such as transistors ${ }^{1,7,15}$, memories ${ }^{16}$, logic circuits ${ }^{17,18}$, light-emitters ${ }^{19}$, and photo-detectors $^{20}$ with flexibility and transparency ${ }^{2,21}$. However, as for any $2 \mathrm{D}$ material, the electrical and optical properties of $\mathrm{MoS}_{2}$ are strongly affected by impurities and its dielectric environment ${ }^{1,2,9,22}$, hindering the study of intrinsic physics and limiting the design of 2Dmaterial-based devices. In particular, the theoretical upper bound of the electron mobility of monolayer (1L) $\mathrm{MoS}_{2}$ is predicted to be from several tens to a few thousands at room temperature $(T)$ and exceed $10^{5} \mathrm{~cm}^{2} / \mathrm{Vs}$ at low $T$ depending on the dielectric environment, impurity density and charge carrier density ${ }^{23-25}$. In contrast, experimentally measured $1 \mathrm{~L} \mathrm{MoS}_{2}$ devices on $\mathrm{SiO}_{2}$ substrates have exhibited room- $T$ two-terminal field-effect mobility that ranges from $0.1-55 \mathrm{~cm}^{2} / \mathrm{Vs}{ }^{1,26,27}$. This value increases to $15-60 \mathrm{~cm}^{2} / \mathrm{Vs}$ with encapsulation by highdielectric materials ${ }^{1,9}$, owing to more effective screening of charged impurities ${ }^{24}$. Due to the presence of large contact resistance from the metal-MoS $\mathrm{S}_{2}$ Schottky barrier, however, these twoterminal measurements underestimate the true channel mobility ${ }^{7,28,29}$. Multi-terminal Hall mobility measurements ${ }^{8,9}$ still show mobility substantially below theoretical limits, particularly at low $T$ with best reported values of $174 \mathrm{~cm}^{2} / \mathrm{Vs}$ at $4 \mathrm{~K}$ for $1 \mathrm{~L}^{9}$ and $250 \mathrm{~cm}^{2} / \mathrm{Vs}$ and $375 \mathrm{~cm}^{2} / \mathrm{Vs}$ at $5 \mathrm{~K}$ for $1 \mathrm{~L}$ and $2 \mathrm{~L}^{8}$. Typically, these thin samples exhibit a crossover to non-metallic behaviour at carrier densities below $\sim 10^{13} \mathrm{~cm}^{-28,9,30}$, or at smaller carrier densities by engineering 
of local defect states and improving interfacial quality ${ }^{31}$. The scattering and disorder that leads to this non-metallic behaviour can arise from multiple origins such as lattice defects, charged impurities in the substrate and surface adsorbates, and it has been difficult to identify their separate contributions ${ }^{1,8,9,23,30-33}$.

\section{van der Waals heterostructure device platform}

We have previously demonstrated that encapsulation of graphene within hBN reduces scattering from substrate phonons and charged impurities, resulting in band transport behaviour that is near the ideal acoustic phonon limit at room $T$, and ballistic over more than $15 \mu \mathrm{m}$ at low $T^{34}$. These results were realized with a novel technique to create one-dimensional edge contacts to graphene exposed by plasma-etching a hBN/graphene/hBN stack. Such an approach has not yet proved effective with $\mathrm{MoS}_{2}$. However, recent reports that graphene can create a high quality electrical contact to $\mathrm{MoS}_{2}{ }^{18,35}$ motivate a hybrid scheme, in which the channel $\mathrm{MoS}_{2}$ and multiple graphene 'leads' are encapsulated in hBN, and the stack is etched to form graphenemetal edge contacts. This new scheme is distinct from previous approaches, in that the entire $\mathrm{MoS}_{2}$ channel is fully encapsulated and protected by $\mathrm{hBN}$, and that we achieve multi-terminal graphene contacts without any contamination from device fabrication process.

Figure 1a and $1 \mathrm{~b}$ show a schematic diagram and optical micrograph of a Hall bar device structure. We employed a 'PDMS (Polydimethylsiloxane) transfer' technique ${ }^{2}$ to place fewlayer graphene flakes around the perimeter of an $\mathrm{MoS}_{2}$ flake, encapsulate them between thicker hBN layers, and place the entire stack on a $\mathrm{Si} / \mathrm{SiO}_{2}$ wafer (Supplementary Fig. 1a). The stack was then shaped into Hall bar geometry such that $\mathrm{hBN}$-encapsulated $\mathrm{MoS}_{2}$ forms the channel. In the contact regions, graphene overlaps the $\mathrm{MoS}_{2}$ and extends to the edge, where it is in turn 
contacted by metal electrodes ${ }^{34}$. Details of the fabrication process are described in the Methods section and Supplementary Information S1. High-resolution scanning transmission electron microscopy (STEM) (Fig. 1c; see also Supplementary Fig. 1b for a larger clean interface area of $>3 \mu \mathrm{m}$ ) confirms that the stacking method can produce ultraclean interfaces free of residue that can be seen when an organic polymer film is used for stacking ${ }^{36}$. We note that while Ohmic contacts have also been achieved in metal- $\mathrm{MoS}_{2}$ contacts by deposition of small work-function metals, vacuum annealing, and electrostatic gating ${ }^{4,17,18}$, top-deposited metal electrodes are not compatible with $\mathrm{hBN}$-encapsulation.

For this study, a series of samples with thickness from 1 - 6 layers $(1 \mathrm{~L}-6 \mathrm{~L})$ was fabricated and measured. The number of layers was identified by Raman and photoluminescence (PL) (see Supplementary Information S2). All samples were obtained by mechanical exfoliation except for the $1 \mathrm{~L}$ sample, for which we used chemical vapor deposition (CVD) grown monolayer $\mathrm{MoS}_{2}$ because of the limited size of mechanically exfoliated monolayers. The CVD-grown $\mathrm{MoS}_{2}$ single crystal has been shown to exhibit high quality from structural, electrical and optical measurements ${ }^{37}$, although the process of transferring it from the growth substrate may introduce more contamination than for mechanically exfoliated flakes.

\section{Gate-tunable graphene- $\mathrm{MoS}_{2}$ contact}

For each sample, we performed temperature-dependent two-probe measurements to examine the quality of the graphene contacts. Figure 2a shows output curves $\left(I_{d s}-V_{d s}\right)$ of a $4 L M_{o} S_{2}$ device at back-gate $\mathrm{V}_{\mathrm{bg}}=80 \mathrm{~V}$. The response is linear at room $T$ and remains linear to low $T$, indicating an Ohmic contact. Similar behaviour is seen for $\mathrm{V}_{\mathrm{bg}}>20 \mathrm{~V}$, whereas gapped behaviour corresponding to non-Ohmic contact is seen for $\mathrm{V}_{\mathrm{bg}}<20 \mathrm{~V}$. This is consistent with 
previous studies which show a gate-tunable contact barrier between graphene and $\mathrm{MoS}_{2}{ }^{18,35}$. In addition, it establishes the gate voltage range over which multi-terminal measurements can be reliably performed. Figure $2 \mathrm{~b}$ shows the measured four-terminal resistivity $\rho$ (in log scale) of the same sample from $\mathrm{V}_{\mathrm{bg}}=20 \mathrm{~V}$ to $80 \mathrm{~V}$ (corresponding to carrier densities of $\sim 4.8 \times 10^{12} \mathrm{~cm}^{-2}$ to $\sim 6.9 \times 10^{12} \mathrm{~cm}^{-2}$, respectively), and from room $T$ to $12 \mathrm{~K} . \rho$ decreases with increasing $\mathrm{V}_{\mathrm{bg}}$, as expected for an $n$-type semiconductor. With decreasing temperature, $\rho$ drops dramatically over the entire accessible range of $\mathrm{V}_{\mathrm{bg}}$, reaching $130 \Omega$ at $12 \mathrm{~K}$. All of the samples studied exhibited similar behaviour: $n$-type semiconducting behaviour and metallic temperature-dependence in the gate voltage accessible to four-terminal measurements.

By comparing the two- and four-terminal results, the contact resistance can be determined (see Supplementary Information S3). The results for the $4 \mathrm{~L} \mathrm{MoS}_{2}$ device, as shown in Fig. 2c, directly demonstrate that the contact resistance can be tuned by back-gate voltage. In fact, a small contact resistance of $\sim 2 \mathrm{k} \Omega \cdot \mu \mathrm{m}$ can be reliably achieved at large gate voltage at room $T$. This likely reflects primarily the graphene- $\mathrm{MoS}_{2}$ junction resistance, since both the graphene resistance and the graphene-metal contact resistance should be substantially less ${ }^{34}$. Below $\mathrm{V}_{\mathrm{bg}}=$ $20 \mathrm{~V}$, the contact resistance increases upon cooling, indicating activated transport across a contact barrier. However, above $\mathrm{V}_{\mathrm{bg}}=20 \mathrm{~V}$, the contact resistance decreases upon cooling, reaching a low- $T$ value of $\sim 1 \mathrm{k} \Omega \cdot \mu \mathrm{m}$ above $\mathrm{V}_{\mathrm{bg}}=50 \mathrm{~V}$. This metallic behaviour directly demonstrates that low-resistance contacts, with no thermal activation, can be achieved at sufficiently high gate voltage. Similar behaviour was observed in all samples (Supplementary Fig. 3), with contact resistance at high $\mathrm{V}_{\mathrm{bg}}$ ranging from $\sim 2-20 \mathrm{k} \Omega \cdot \mu \mathrm{m}$ at $\operatorname{room} T$ and $\sim 0.7-10$ $\mathrm{k} \Omega \cdot \mu \mathrm{m}$ at low $T$. These values are comparable to room- $T$ values reported previously for graphene 38 and metal ${ }^{39-41}$ contacts, but larger than the best contacts achieved by $\mathrm{MoS}_{2}$ phase engineering 
$(0.2-0.3 \mathrm{k} \Omega \cdot \mu \mathrm{m})^{29}$. Due to the increase in band gap with decreasing thickness, the value of $\mathrm{V}_{\mathrm{bg}}$ required to achieve Ohmic contact is larger for thinner samples.

\section{Scattering mechanism in $\mathrm{MoS}_{2}$}

To examine the quality of the hBN-encapsulated devices and determine the scattering mechanisms limiting the carrier mobility of $\mathrm{MoS}_{2}$, the Hall mobility $\mu_{\text {Hall }}(T)$ was derived from $\rho(T)$ and the carrier density $n\left(\mathrm{~V}_{\mathrm{bg}}\right)$ (obtained by Hall effect measurements, see Supplementary Information S4). Figure 3a shows $\mu_{\mathrm{Hall}}$ for the $1 \mathrm{~L}-6 \mathrm{~L}$ samples as a function of temperature, at carrier densities varying from $4.0 \times 10^{12} \mathrm{~cm}^{-2}$ to $1.2 \times 10^{13} \mathrm{~cm}^{-2}$ (see Fig. $3 \mathrm{~b}$ and Supplementary Table 1). Thinner samples were measured at higher carrier densities required to achieve Ohmic contacts. For all of the samples, mobility increases with decreasing temperature and saturates at a constant value at low $T$. The low- $T$ mobility in our devices is much higher than previously reported values, and there is no sign of metal-insulator transition as observed at similar carrier densities around $10^{13} \mathrm{~cm}^{-2}$ in $\mathrm{SiO}_{2}$-supported $\mathrm{MoS}_{2}{ }^{8,9,30,32,33}$. This strongly suggests that extrinsic scattering and disorder (either from $\mathrm{SiO}_{2}$ or from processing with polymer resists) has been the primary source of non-metallic behaviour in $\mathrm{MoS}_{2}$ measured to date.

The measured mobility curves can be reasonably fitted to a simple functional form: $\frac{1}{\mu(T)}=\frac{1}{\mu_{i m p}}+\frac{1}{\mu_{p h}(T)}$, where $\mu_{\mathrm{imp}}$ is the contribution from impurity scattering, and $\mu_{\mathrm{ph}}$ is the temperature-dependent contribution due to phonon scattering. In all samples, the fitted $\mu_{\mathrm{ph}}(T)$ is well described by a power law $\mu_{\mathrm{ph}} \sim \mathrm{T}^{-\gamma}$ above $100 \mathrm{~K}$ (Supplementary Fig. 7). This behaviour is consistent with mobility limited by $\mathrm{MoS}_{2}$ optical phonons, as theoretically predicted to have an exponent of $\sim 1.69$ in monolayer ${ }^{23}$ and $\sim 2.5$ for bulk $\mathrm{MoS}_{2}{ }^{42}$ at $\mathrm{T}>100 \mathrm{~K}$. Although this power 
law behaviour has been observed in experiments by other groups ${ }^{8,9,30}$, a stronger temperature dependence was observed in our devices, with the exponent $\gamma$ ranging from 1.9 - 2.5 (inset table of Fig. 3a), as opposed to $0.55-1.7$ reported previously ${ }^{8,9,30}$. We also note that the room- $T$ mobility, which is dominated by phonon scattering in all of the samples, is seen to vary from 40 $120 \mathrm{~cm}^{2} /$ Vs. At this point we can find no satisfactory explanation for this variation: there is no discernible trend with thickness, and no variation of the gamma value with carrier density (see Supplementary Fig. 8). Finally, we note that a deviation from the simple form $\mu_{\mathrm{ph}} \sim \mathrm{T}^{-\gamma}$ in high mobility samples below $100 \mathrm{~K}$ may indicate acoustic phonon scattering, although further study is needed to fully explore this regime.

At the lowest temperature, phonon scattering is suppressed, and the residual resistivity is due to dominant long-ranged Coulomb impurities or short-ranged atomic defects ${ }^{43-45}$, captured in the measured quantity $\mu_{\mathrm{imp}}$. Figure $3 \mathrm{~b}$ shows the derived values of $\mu_{\mathrm{imp}}$ as a function of carrier density $n$. For each sample, $\mu_{\text {imp }}$ increases with $n$, with maximum values ranging from 1,020 $\mathrm{cm}^{2} / \mathrm{Vs}$ in the CVD monolayer to $34,000 \mathrm{~cm}^{2} / \mathrm{Vs}$ for $6 \mathrm{~L}$, up to two orders of magnitude higher than previously reported values ${ }^{8,46}$ (Fig. $3 \mathrm{~b}$ and Supplementary Table 1). These basic trends allow us to rule out scattering due to impurities or defects located within the $\mathrm{MoS}_{2}$ itself: bulk charged impurities should give rise to thickness-independent mobility, and short-ranged scatterers due to atomic defects should give rise to a density-independent mobility ${ }^{45}$. On the other hand, interfacial scatterers, including both Coulomb impurities and short-ranged defects, which are limiting scattering mechanisms in high quality conventional 2D electron gas system ${ }^{44}$ are promising candidates. Indeed, PDMS transfer, while substantially cleaner than methods involving organic polymers, can potentially introduce adsorbates to the top $\mathrm{MoS}_{2}$ surface. 
To understand the effects of interfacial scattering on samples with different thickness, we model interfacial Coulomb and short-range scattering as a function of carrier density, for samples from $1 \mathrm{~L}$ to $6 \mathrm{~L}$ in thickness. For Coulomb scattering, we employed a model based on a perturbative approach by Stern ${ }^{47}$, from which we obtained the screened Coulomb potential used in the mobility calculation. This model has also been commonly used in the context of semiconductor devices (see Supplementary Information S8). Within the model, increasing carrier density enhances screening of the interfacial Coulomb potential, which leads to improved carrier mobility, and increasing the thickness of $\mathrm{MoS}_{2}$ redistributes the charge centroid further from the interface, also resulting in enhancement of mobility. The calculated mobility is shown in Fig. 3c, assuming the same impurities concentration of $6 \times 10^{9} \mathrm{~cm}^{-2}$ (chosen to match the $6 \mathrm{~L}$ data) located at the top $\mathrm{MoS}_{2}$ interface across devices with different number of layers. Although the qualitative trend of increasing mobility with layer numbers and carrier densities is consistent with the model, the model fails to account for the observed large thickness dependence of more than an order of magnitude between the $1 \mathrm{~L}$ and $6 \mathrm{~L}$ devices. The changes in the model calculations including the effects of band structure change with increasing layer thickness is discussed in Supplementary Information S8.

We next consider interfacial short-ranged scatterers with atomically localized scattering potentials, which can be modeled as delta-function potentials within the same framework as used above. Quantum lifetime measurements, to be discussed later, suggest that these scatterers strongly dominate electronic transport in the $1 \mathrm{~L}$ devices. We therefore set the interfacial shortranged scattering parameter (the product of scattering potential and defect density, see Supplementary Information S8) to fit the mobility of the 1L device. In this case, for the same 
interfacial scattering the mobility increases rapidly with sample thickness - much more than observed experimentally.

Based on this analysis, we propose that the interfaces in our devices introduce both longranged Coulomb scattering and short-ranged scattering. In this case, we can calculate the total extrinsic mobility using Matthiessen's rule. The combination of long-ranged and short-ranged scatterers provides a better agreement to the observed layer-dependent mobility as shown in Fig. 3e, a salient point which we will revisit again later in quantum oscillations study. Of course, a perfect match to experiment is not expected due to sample-to-sample variation in impurity density. We also note that the long-ranged impurity density of $6 \times 10^{9} \mathrm{~cm}^{-2}$ is two orders of magnitude smaller than typically obtained for graphene on $\mathrm{SiO}_{2}$, and hence accounts for the two orders of magnitude larger mobility we obtained as compared to the best reported devices ${ }^{8,46}$.

\section{Observation of Shubnikov-de Haas (SdH) oscillations in $\mathrm{MoS}_{2}$}

Figure 4 shows the longitudinal $\left(R_{x x}\right)$ and Hall resistance $\left(R_{x y}\right)$ of the monolayer (Fig. 4a), 4L $\mathrm{MoS}_{2}$ (Fig. 4b) and 6L MoS 2 (Fig. 4c) samples as a function of applied magnetic field. We observe pronounced $\mathrm{SdH}$ oscillations in $\mathrm{MoS}_{2}$ for the first time, providing additional strong evidence of high quality and homogeneity in the heterostructure devices. In the highest-mobility (6L) sample (Fig. 4c), the onset of $\mathrm{SdH}$ oscillations is close to 1 Tesla (T), further confirming its ultra-high mobility. Encouragingly, the high-field Hall resistance (blue curve, $R_{x y}$ ) begins to reveal plateau-like structures at high magnetic fields coinciding with $R_{x x}$ minima. These emerging features were similarly observed in early studies of graphene samples with moderate mobility ${ }^{48}$, giving hope that fully developed quantum Hall states can be observed with further improvements in sample quality. The periodicity of the $\mathrm{SdH}$ oscillations can be used to estimate 
the carrier density, or equivalently to measure the level degeneracy $g$ for a known density (for details see Supplementary S7). For the $1 \mathrm{~L}$ samples, we observe $2<g<4$, indicating that the bands may be partially valley-spin split (the multi-band nature of the $6 \mathrm{~L}$ sample complicates this analysis). This is consistent with extra $\mathrm{SdH}$ oscillations that begin to emerge at high fields, but more detailed study is required to explore this splitting in detail.

The quantum scattering time $\tau_{\mathrm{q}}$ which is limited by both small and large angle scattering that destroys quantized cyclotron orbits, can be estimated from the magnetic field corresponding to the onset of $\mathrm{SdH}$ oscillations, following the relation $\mu_{\mathrm{q}}=e \tau_{q} / m^{*} \sim 1 / B_{\mathrm{q}}{ }^{49}$, where $e$ is the electron charge and $m^{*}$ is the effective mass obtained from ab initio bandstructure calculations ${ }^{50}$. This yields quantum mobilities for $1 \mathrm{~L}, 4 \mathrm{~L}$ and $6 \mathrm{~L} \mathrm{MoS}_{2}$ of $\sim 1,400 \mathrm{~cm}^{2} / \mathrm{Vs}, \sim 3,100 \mathrm{~cm}^{2} / \mathrm{Vs}$ and $\sim$ $10,000 \mathrm{~cm}^{2} / \mathrm{Vs}$, respectively. A more accurate estimate of $\tau_{\mathrm{q}}$ in $\mathrm{MoS}_{2}$ can be obtained using a Dingle plot (Supplementary Fig. 9), a well-established method in conventional 2D electron gas systems (2DEGs) (for details see Supplementary S6). The inset of Fig. 4a shows SdH oscillations of $1 \mathrm{~L} \mathrm{MoS}$, after subtraction of a magnetoresistance background, as function of $1 / B$. The red dashed line is the fitted envelope, from which we estimate a quantum scattering time of $\tau_{\mathrm{q}}=176$ fs. We show the values of $\tau_{\mathrm{q}}$ obtained using both methods (oscillation onset and Dingle plots) in Supplementary Fig. 9c. The ratio of transport to quantum scattering time can provide additional evidence of predominant scattering sources. In our samples a ratio near 1 in $1 \mathrm{~L}$ devices indicates predominantly short-ranged scattering, and an increase in $\tau_{\mathrm{t}} / \tau_{\mathrm{q}}$ with increasing thickness indicates a crossover to long-range scattering. This trend consistent with our previous physical picture of low- $T$ electronic transport dominated by a mix of short- and long-ranged interfacial impurities.

\section{Conclusion}


We demonstrate a vdW heterostructure device platform in which an atomically thin $\mathrm{MoS}_{2}$ layer is encapsulated by $\mathrm{hBN}$ and contacted by graphene. The vdW heterostructure provides a standard device platform that enables us to measure intrinsic electrical transport of $2 \mathrm{D}$ materials and achieve high mobility 2D devices for studying the unique transport properties and novel quantum physics. By forming robust and tunable electrical contacts and dramatically reducing interfacial impurities, intrinsic electron-phonon scattering can be observed at high $T$, and substantially improved mobility can be achieved at low $T$. This enables the first observation of Shubnikov-de Haas oscillations in $\mathrm{MoS}_{2}$. Modeling and quantum lifetime analysis suggest that a combination of short-ranged and long-ranged interfacial scattering limits the low- $T$ mobility, indicating that further improvements should be possible.

\section{Methods}

Device fabrication. The $\mathrm{hBN} / \mathrm{MoS}_{2} /$ graphene/hBN stacks were fabricated using the 'PDMS transfer' $^{2}$ technique on $285 \mathrm{~nm} \mathrm{SiO}_{2} / \mathrm{Si}$ substrates. The transfer techniques are described in detail in the Supplementary Information S1. The stacks were then shaped to the desired Hall bar structure through electron-beam patterning and reactive ion etching (RIE) with a mixture of $\mathrm{CHF}_{3}$ and $\mathrm{O}_{2}$. Finally, metal leads were patterned by e-beam lithography and subsequent deposition of metals $(\mathrm{Cr} 1 \mathrm{~nm} / \mathrm{Pd} 20 \mathrm{~nm} / \mathrm{Au} 50 \mathrm{~nm})$. The metal leads make edge-contact to graphene electrodes as reported previously ${ }^{34}$.

TEM sample preparation. For high-resolution imaging, we fabricated a cross-sectional TEM lift-out sample from the finished encapsulated devices, using a FEI Strata 400 dual-beam Focused Ion Beam. STEM imaging was conducted in a FEI Tecnai F-20 STEM operated at 
$200 \mathrm{kV}$, with a $9.6 \mathrm{mrad}$ convergence semiangle and high-angle annular dark field detector. False coloring was added by hand.

Electrical measurements and magneto-transport measurements. Two-terminal transport characteristics were measured by applying DC bias (Keithley 2400) to the source and gate electrodes and measuring the drain current using a current amplifier (DL 1211). For fourterminal measurements, a standard lock-in amplifier (SR830) measured voltage drop across the channel with constant current bias. Magneto-transport measurements were performed in a Physical Property Measurement System (PPMS) (Fig. 4c) and a $\mathrm{He}_{3}$ cryostat at the National High Magnetic Field Laboratory (NHMFL) (Fig. 4a and b).

\section{References}

1. Radisavljevic, B., Radenovic, A., Brivio, J., Giacometti, V. \& Kis, A. Single-layer $\mathrm{MoS}_{2}$ transistors. Nature Nanotech. 6, 147-150 (2011).

2. Lee, GH. et al. Flexible and transparent $\mathrm{MoS}_{2}$ field-effect transistors on hexagonal boron nitride-graphene heterostructures. ACS Nano 7, 7931-7936 (2013).

3. Mak, K., Lee, C., Hone, J., Shan, J. \& Heinz, T. Atomically Thin MoS 2 : A New DirectGap Semiconductor. Phy. Rev. Lett. 105, 136805 (2010).

4. Lee, C. H. et al. Atomically thin $\mathrm{p}-\mathrm{n}$ junctions with van der Waals heterointerfaces. Nature Nanotech. 9, 676-681 (2014).

5. Lee, C. et al. Anomalous lattice vibrations of single-and few-layer $\mathrm{MoS}_{2}$. ACS Nano 4, 2695-2700 (2010).

6. Kim, S. et al. High-mobility and low-power thin-film transistors based on multilayer $\mathrm{MoS}_{2}$ crystals. Nature Commun. 3, 1011 (2012).

7. Das, S., Chen, H.-Y., Penumatcha, A. \& Appenzeller, J. High Performance Multilayer $\mathrm{MoS}_{2}$ with Scandium Contacts. Nano Lett. 13, 100-105 (2013).

8. Baugher, B., Churchill, H., Yang, Y. \& Jarillo-Herrero, P. Intrinsic Electronic Transport Properties of High-Quality Monolayer and Bilayer $\mathrm{MoS}_{2}$. Nano Lett. 13, 4212-4216 (2013). 9. Radisavljevic, B. \& Kis, A. Mobility engineering and a metal-insulator transition in monolayer $\mathrm{MoS}_{2}$. Nature Mater. 12, 815-820 (2013).

10. Xiao, D., Liu, G.-B., Feng, W., Xu, X. \& Yao, W. Coupled Spin and Valley Physics in Monolayers of $\mathrm{MoS}_{2}$ and Other Group-VI Dichalcogenides. Phys. Rev. Lett 108, 196802 (2012). 11. Mak, K., McGill, K., Park, J. \& McEuen, P. Valleytronics. The valley Hall effect in $\mathrm{MoS}_{2}$ transistors. Science 344, 1489-1492 (2014). 
12. Zeng, H., Dai, J., Yao, W., Xiao, D. \& Cui, X. Valley polarization in $\mathrm{MoS}_{2}$ monolayers by optical pumping. Nature Nanotech. 7, 490-493 (2012).

13. Jiang, T. et al. Valley and band structure engineering of folded $\mathrm{MoS}_{2}$ bilayers. Nature Nanotech. 9, 825-829 (2014).

14. Mak, K., He, K., Shan, J. \& Heinz, T. Control of valley polarization in monolayer $\mathrm{MoS}_{2}$ by optical helicity. Nature Nanotech. 7, 494-498 (2012).

15. Bao, W., Cai, X., Kim, D., Sridhara, K. \& Fuhrer, M. High mobility ambipolar $\mathrm{MoS}_{2}$ field-effect transistors: Substrate and dielectric effects. Applied Physics Letters 102, 042104 (2013).

16. Choi, M., Lee, GH., Yu, Y., Lee, D. \& Lee, S. Controlled charge trapping by molybdenum disulphide and graphene in ultrathin heterostructured memory devices. Nature Commun. 4, 1642 (2013).

17. Wang, H. et al. Integrated circuits based on bilayer $\mathrm{MoS}_{2}$ transistors. Nano Lett. 12, 4674-4680 (2012).

18. Yu, L. et al. Graphene/ $\mathrm{MoS}_{2}$ Hybrid Technology for Large-Scale Two-Dimensional Electronics. Nano Lett. 14, 3055-3063 (2014).

19. Sundaram, R., Engel, M., Lombardo, A. \& Krupke, R. Electroluminescence in single layer $\mathrm{MoS}_{2}$. Nano Lett. 13, 1416-1421 (2013).

20. Britnell, L. et al. Strong Light-Matter Interactions in Heterostructures of Atomically Thin Films. Science 340, 1311-1314 (2013).

21. Yoon, J. et al. Highly Flexible and Transparent Multilayer $\mathrm{MoS}_{2}$ Transistors with Graphene Electrodes. Small 9, 3295-3300 (2013).

22. Qiu, H. et al. Electrical characterization of back-gated bi-layer $\mathrm{MoS}_{2}$ field-effect transistors and the effect of ambient on their performances. Appl. Rhys. Lett. 100, 123104 (2012). 23. Kaasbjerg, K., Thygesen, K. \& Jacobsen, K. Phonon-limited mobility in n-type singlelayer $\mathrm{MoS}_{2}$ from first principles. Phy. Rev. B 85, 115317 (2012).

24. Ma, N. \& Jena, D. Charge Scattering and Mobility in Atomically Thin Semiconductors. Phys. Rev. X 4, 011043 (2014).

25. Li, X. et al. Intrinsic electrical transport properties of monolayer silicene and $\mathrm{MoS}_{2}$ from first principles. Phys. Rev. B 87, 115418 (2013).

26. Novoselov, K. et al. Two-dimensional atomic crystals. Proceedings of the National Academy of Sciences of the United States of America 102, 10451-10453 (2005).

27. Kappera, R, Voiry, D, Yalcin, SE, Jen, W \& Acerce, M. Metallic 1T phase source/drain electrodes for field effect transistors from chemical vapor deposited $\mathrm{MoS}_{2}$. Appl. Rhys. Lett. 2, 092516 (2014).

28. Guo, Y. et al. Study on the Resistance Distribution at the Contact between Molybdenum Disulfide and Metals. ACS Nano 8, 7771-7779 (2014).

29. Kappera, R. et al. Phase-engineered low-resistance contacts for ultrathin $\mathrm{MoS}_{2}$ transistors. Nature Mater. 13, 1128-1134 (2014).

30. Schmidt, H. et al. Transport Properties of Monolayer $\mathrm{MoS}_{2}$ Grown by Chemical Vapor Deposition. Nano Letters 14, 1909-1913 (2014).

31. Yu, Z. et al. Towards intrinsic charge transport in monolayer molybdenum disulfide by defect and interface engineering. Nature Commun. 5, 5290 (2014).

32. Zhu, W. et al. Electronic transport and device prospects of monolayer molybdenum disulphide grown by chemical vapour deposition. Nature Commun. 5, 3078 (2014).

33. Qiu, H. et al. Hopping transport through defect-induced localized states in molybdenum 
disulphide. Nature Commun. 4, 2642 (2013)

34. Wang, L. et al. One-Dimensional Electrical Contact to a Two-Dimensional Material.

Science 342, 614-617 (2013).

35. Roy, T. et al. Field-Effect Transistors Built from All Two-Dimensional Material

Components. ACS Nano 8, 6256-6264 (2014).

36. Haigh, S., Gholinia, A., Jalil, R., Romani, S. \& Britnell, L. Cross-sectional imaging of individual layers and buried interfaces of graphene-based heterostructures and superlattices.

Nature Mater. 11, 764-767 (2012).

37. Zande, AM van der et al. Grains and grain boundaries in highly crystalline monolayer molybdenum disulphide. Nature Mater. 12, 554-561 (2013).

38. Du, Y., Yang, L., Liu, H. \& Ye, P. Contact research strategy for emerging molybdenum disulfide and other two-dimensional field-effect transistors. APL Materials 2, 092510 (2014).

39. Das, S. \& Appenzeller, J. Where does the current flow in two-dimensional layered systems? Nano Lett. 13, 3396-3402 (2013).

40. Liu, H., Neal, A. T. \& Ye, P. D. Channel length scaling of $\mathrm{MoS}_{2}$ MOSFETs. ACS Nano 6, 8563-8569 (2012).

41. Liu, H. et al. Switching Mechanism in Single-Layer Molybdenum Disulfide Transistors: An Insight into Current Flow across Schottky Barriers. ACS Nano 8, 1031-1038 (2013).

42. Fivaz, R. \& Mooser, E. Mobility of Charge Carriers in Semiconducting Layer Structures. Phys. Rev. 163, 743755 (1967).

43. Chen, J.-H., Jang, C., Xiao, S., Ishigami, M. \& Fuhrer, M. S. Intrinsic and extrinsic performance limits of graphene devices on $\mathrm{SiO}_{2}$. Nature Nanotech. 3, 206-209 (2008).

44. Ando, T., Fowler, A. B. \& Stern, F. Electronic properties of two-dimensional systems. Rev. Mod. Phys. 54, 437 (1982).

45. Sarma, D., Adam, S., Hwang, E. \& Rossi, E. Electronic transport in two-dimensional graphene. Rev. Mod. Phys. 83, 407 (2011).

46. Neal, A., Liu, H., Gu, J. \& Ye, P. Magneto-transport in $\mathrm{MoS}_{2}$ : phase coherence, spinorbit scattering, and the hall factor. ACS Nano 7, 7077-1082 (2013).

47. Stern, F. \& Howad W. E. Properties of Semiconductor Surface Inversion Layers in the Electric Quantum Limit. Phys. Rev.163, 816. (1967)

48. Novoselov, K. et al. Electric Field Effect in Atomically Thin Carbon Films. Science 306, 666-669 (2004).

49. Kretinin, A. V. et al. Electronic properties of graphene encapsulated with different twodimensional atomic crystals. Nano Lett. 14, 3270-3276 (2014).

50. Liu, G.-B., Shan, W.-Y., Yao, Y., Yao, W. \& Xiao, D. Three-band tight-binding model for monolayers of group-VIB transition metal dichalcogenides. Physical Review B 88, 085433 (2013). 


\section{Acknowledgements}

This research was supported by the U.S. National Science Foundation (DMR-1122594), the NSF MRSEC program through Columbia in the Center for Precision Assembly of Superstratic and Superatomic Solids (DMR-1420634), and in part by the FAME Center, one of six centers of STARnet, a Semiconductor Research Corporation program sponsored by MARCO and DARPA. G.H.L was supported by Basic Science Research Program (NRF-2014R1A1A1004632) through the National Research Foundation (NRF) funded by the Korean government Ministry of Science, ICT and Future Planning, and in part by the Yonsei University Future-leading Research Initiative of 2014. P.Y.H. acknowledges support from the NSF Graduate Research Fellowship Program under grant DGE-0707428. Additional support was provide through funding and shared facilities from the Cornell Center for Materials Research NSF MRSEC program (DMR-1120296). F.P. and B.S.J. acknowledged the Center for Nanostructured Graphene (CNG), which is funded by the Danish National Research Foundation, Project DNRF58. K.W. and T.T. acknowledge support from the Elemental Strategy Initiative conducted by the MEXT, Japan. T.T. acknowledges support a Grant-in-Aid for Scientific Research on Grant 262480621 and on Innovative Areas "NanoInformatics" (Grant 25106006) from JSPS. The high magnetic field measurements were performed at the NHMFL and the authors thank Alexey Suslov, Bobby Joe Pullum, Jonathan Billings, and Tim Murphy for assistance with the experiments at NHMFL.

\section{Author Contributions}

X.C. and G.H.L. designed the research project and supervised the experiment. X.C., G.H.L., Y.D.K., G.A., C.H.L., F.Y., F.P., B.S.J., and L.W. performed device fabrication and X.C., 
G.H.L. and Y.D.K. performed device measurements under supervision of P.K. and J.H.. X.C., G.H.L., G.A., X.Z. performed optical spectroscopy and data analysis. D.A.C. grew and prepared the CVD $\mathrm{MoS}_{2}$ sample. T.L. performed the theoretical calculations. K.W. and T.T. prepared hBN samples. P.Y.H. and D.A.M. performed TEM analyses. X.C., G.H.L., Y.D.K. and J.H. analyzed the data and wrote the paper.

\section{Figure Captions}

Figure 1 | vdW device structure and interface characterization. a, Schematic of the hBNencapsulated $\mathrm{MoS}_{2}$ multi-terminal device. Exploded view shows the individual components that constitute the heterostructure stack. The bottom panel shows the zoom-in cross-sectional schematic of metal-graphene- $\mathrm{MoS}_{2}$ contact region. b, Optical microscope image of a fabricated device. Graphene contact regions are outlined by dashed lines. c, Cross-section STEM image of the fabricated device. The zoom-in false-color image clearly shows the ultra-sharp interfaces between different layers. (graphene: 5L, $\mathrm{MoS}_{2}: 3 \mathrm{~L}$, top-hBN: $8 \mathrm{~nm}$, bottom-hBN: $19 \mathrm{~nm}$ )

Figure 2 | Gate-tunable and temperature-dependent graphene-MoS $\mathrm{S}_{2}$ contact. a, Output curves $\left(\mathrm{I}_{\mathrm{ds}}-\mathrm{V}_{\mathrm{ds}}\right)$ of the $\mathrm{hBN}$-encapsulated $4 \mathrm{~L} \mathrm{MoS}$ device with graphene electrodes at varying temperature. The back gate voltage $\left(\mathrm{V}_{\mathrm{bg}}\right)$ is kept at $80 \mathrm{~V}$ with carrier density of $6.85 \times 10^{12} \mathrm{~cm}^{-2}$ in $\mathrm{MoS}_{2}$. The linearity of output curves confirms that graphene- $\mathrm{MoS}_{2}$ contact is Ohmic at all temperatures. b, Resistivity ( $\rho$ ) of $4 \mathrm{~L} \mathrm{MoS}_{2}$ (log scale) as a function of $\mathrm{V}_{\mathrm{bg}}$ at varying temperature. The resistivity decreases upon cooling, showing metallic behaviour, reaching $\sim 130$

$\Omega$ at $12 \mathrm{~K}$. c, Contact resistance $\left(\mathrm{R}_{\mathrm{C}}\right)$ of the same device (log scale) as a function of $\mathrm{V}_{\mathrm{bg}}$ at varying temperature. The inset shows the $\mathrm{R}_{\mathrm{C}}$ as a function of temperature at different $\mathrm{V}_{\mathrm{bg}}$. At high $\mathrm{V}_{\mathrm{bg}}$, contact resistance even decreases when decreasing the temperature. 


\section{Figure 3 | Temperature, carrier density dependence of Hall mobility and scattering}

mechanism. a, Hall mobility of hBN-encapsulated $\mathrm{MoS}_{2}$ devices with different number of layers of $\mathrm{MoS}_{2}$ as a function of temperature. To maintain Ohmic contacts, a finite $\mathrm{V}_{\mathrm{bg}}$ was applied. The measured carrier densities from Hall measurements for each device are listed in Supplementary Table 1. The solid fitting lines are drawn by the model in the main text. All the fitting parameters are listed in Supplementary Table 1 . For a visual guideline, a dashed line of power law $\mu_{\mathrm{ph}} \sim \mathrm{T}^{-\gamma}$ is drawn and fitted values of $\gamma$ for each device are listed in the inset table. $\mathbf{b}$, Impurity-limited mobility $\left(\mu_{\mathrm{imp}}\right)$ as a function of carrier density of $\mathrm{MoS}_{2}$. For comparison, the previously reported values from $\mathrm{MoS}_{2}$ on $\mathrm{SiO}_{2}$ substrates (Ref. 8, 46) are plotted. c to e, The solid lines show the theoretically calculated long-ranged (LR) impurity limited mobility (c), short-ranged (SR) impurity limited mobility (d) and mobility including both LR and SR based on Matthiessen's rule $1 / \mu=1 / \mu_{\mathrm{LR}}+1 / \mu_{\mathrm{SR}}$ as a function of carrier density for $1 \mathrm{~L}$ to $6 \mathrm{~L} \mathrm{MoS}_{2}(\mathrm{e})$. The experimental data from $1 \mathrm{~L}$ and $6 \mathrm{~L}$ are shown in dots (c-e).

Figure 4 | Observation of Shubnikov-de Haas oscillations in hBN-encapsulated $\mathrm{MoS}_{2}$ device. a, Longitudinal resistance $R_{x x}$ (red curve) and Hall resistance $R_{x y}$ (blue curve) of hBNencapsulated CVD 1L MoS 2 device as a function of magnetic field $(B)$ measured at $0.3 \mathrm{~K}$ and carrier density of $9.7 \times 10^{12} \mathrm{~cm}^{-2}$. The inset shows the oscillation amplitude (black curve) as a function of 1/B after subtraction of the magneto-resistance background. The quantum scattering time extracted from the fitted Dingle plot (red dashed line) is $176 \mathrm{fs} . \mathbf{b}, R_{x x}$ (red curve) and $R_{x y}$ (blue curve) of $\mathrm{hBN}$-encapsulated $4 \mathrm{~L} \mathrm{MoS}_{2}$ device as a function of $B$. Hall measurement was conducted at $0.3 \mathrm{~K}$ and carrier density of $4.9 \times 10^{12} \mathrm{~cm}^{-2}$. c, $R_{x x}$ (red curve) and $R_{x y}$ (blue curve) of hBN-encapsulated $6 \mathrm{~L} \mathrm{MoS}_{2}$ device as a function of $B$. Hall measurement was conducted at 3 $\mathrm{K}$ and carrier density of $5.3 \times 10^{12} \mathrm{~cm}^{-2}$. 

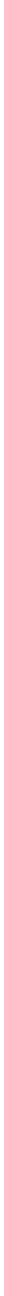

C

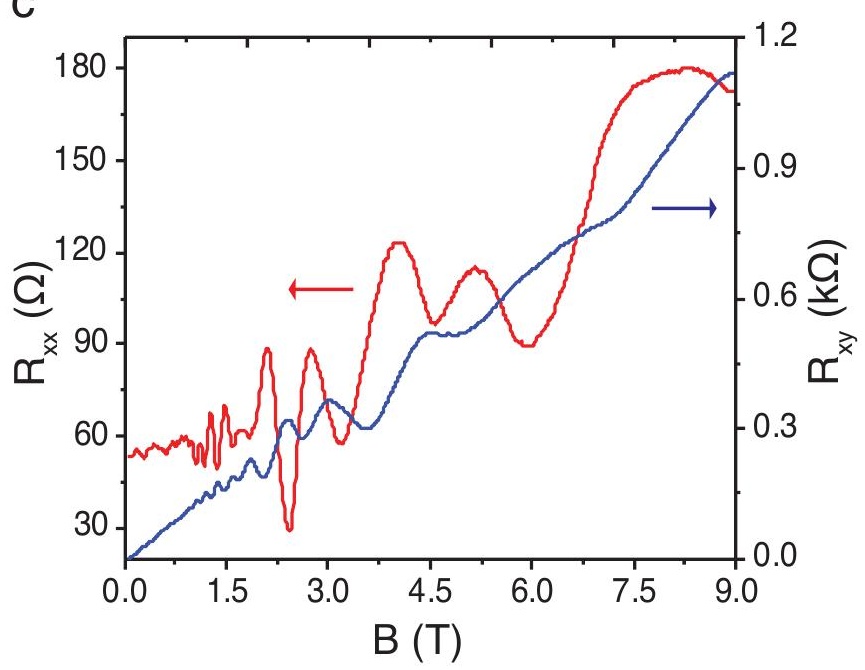




\section{Supplementary Information for}

Multi-terminal transport measurements of $\mathrm{MoS}_{2}$ using a van der Waals heterostructure device platform

Xu Cui, Gwan-Hyoung Lee, Young Duck Kim, Ghidewon Arefe, Pinshane Y. Huang, Chul-Ho

Lee, Daniel A. Chenet, Xian Zhang, Lei Wang, Fan Ye, Filippo Pizzocchero, Bjarke S. Jessen, Kenji Watanabe, Takashi Taniguchi, David A. Muller, Tony Low, Philip Kim, and James Hone 


\section{Experimental}

S1. Fabrication of hBN-encapsulated $\mathrm{MoS}_{2}$ devices

S2. Identification of $\mathrm{MoS}_{2}$ flakes on PDMS

S3. Contact resistance and Schottky barrier of graphene-MoS $\mathrm{M}_{2}$ contacts

S4. Hall measurements and Hall mobility calculations

S5. Optical phonon-limited mobility in $\mathrm{MoS}_{2}$

S6. Quantum scattering time analysis in $\mathrm{MoS}_{2}$

S7. Periodicity analysis of SdH oscillations in monolayer $\mathrm{MoS}_{2}$

Modeling

S8. Modeling $\mathrm{MoS}_{2}$ electron mobility limited by interfacial Coulomb impurities and shortranged defects 


\section{S1. Fabrication of hBN-encapsulated $\mathrm{MoS}_{2}$ devices}

As explained in our previous report ${ }^{1}$, we directly exfoliated hexagonal boron nitride (hBN), $\mathrm{MoS}_{2}$, and graphene flakes onto Polydimethylsiloxane (PDMS) stamps using mechanical exfoliation with Scotch tape and conducted multiple transfers onto $\mathrm{hBN}$ on a $\mathrm{SiO}_{2}$ substrate. First, $\mathrm{hBN}$ flakes with a thickness of $10-30 \mathrm{~nm}$ were mechanically exfoliated onto $285 \mathrm{~nm} \mathrm{SiO}_{2} / \mathrm{Si}$ chips. Then, $\mathrm{MoS}_{2}$ (SPI Supplies) and few-layer graphene (Covalent Materials Co.) were separately prepared on PDMS stamps. The $\mathrm{MoS}_{2}$ flake on the PDMS stamp was inverted and aligned onto the target $\mathrm{hBN}$ flake on the $\mathrm{SiO}_{2}$ substrate by micro-manipulator and held in contact for 5 minutes at $40{ }^{\circ} \mathrm{C}$ in order to transfer the flake to the substrate. Next, graphene from PDMS was transferred to the $\mathrm{hBN} / \mathrm{MoS}_{2}$ stack and positioned to serve as electrodes. This step was repeated multiple times to place graphene electrodes around the perimeter of the $\mathrm{MoS}_{2}$ channel. Finally, another hBN flake on PDMS was transferred on top of the $\mathrm{hBN} / \mathrm{MoS}_{2} /$ graphene stack to provide complete encapsulation of the $\mathrm{MoS}_{2}$ channel. The $\mathrm{hBN} /$ graphene/MoS$/ \mathrm{hBN}$ stack was formed sequentially as shown in Fig. S1a (i-vii). We used e-beam lithography to shape the stack of hBN/graphene/MoS $/$ hBN into a Hall bar geometry using patterned Poly(methyl methacrylate) (PMMA) as an etch mask as shown in Fig. S1a (viii). We performed a dry etching process using inductively coupled plasma (ICP, Oxford 80) with a mixture of $\mathrm{CHF}_{3}$ and $\mathrm{O}_{2}$ gas. The stack was etched until the edges of the graphene flakes were exposed (Fig. S1a (ix)). After dissolving the PMMA film in acetone, a second e-beam patterning was followed to define the metal leads. Metal leads of $\mathrm{Cr}(1 \mathrm{~nm}) / \mathrm{Pd}(20 \mathrm{~nm}) / \mathrm{Au}(50 \mathrm{~nm})$ was deposited by e-beam evaporation as shown in Fig. S1a(x). Ohmic contact is formed along edges of graphene between metal and graphene ${ }^{2}$. A cross-section STEM image in Fig. S1b confirms that the multi-stacked heterostructure with clean heterointerfaces can be achieved by this method. 

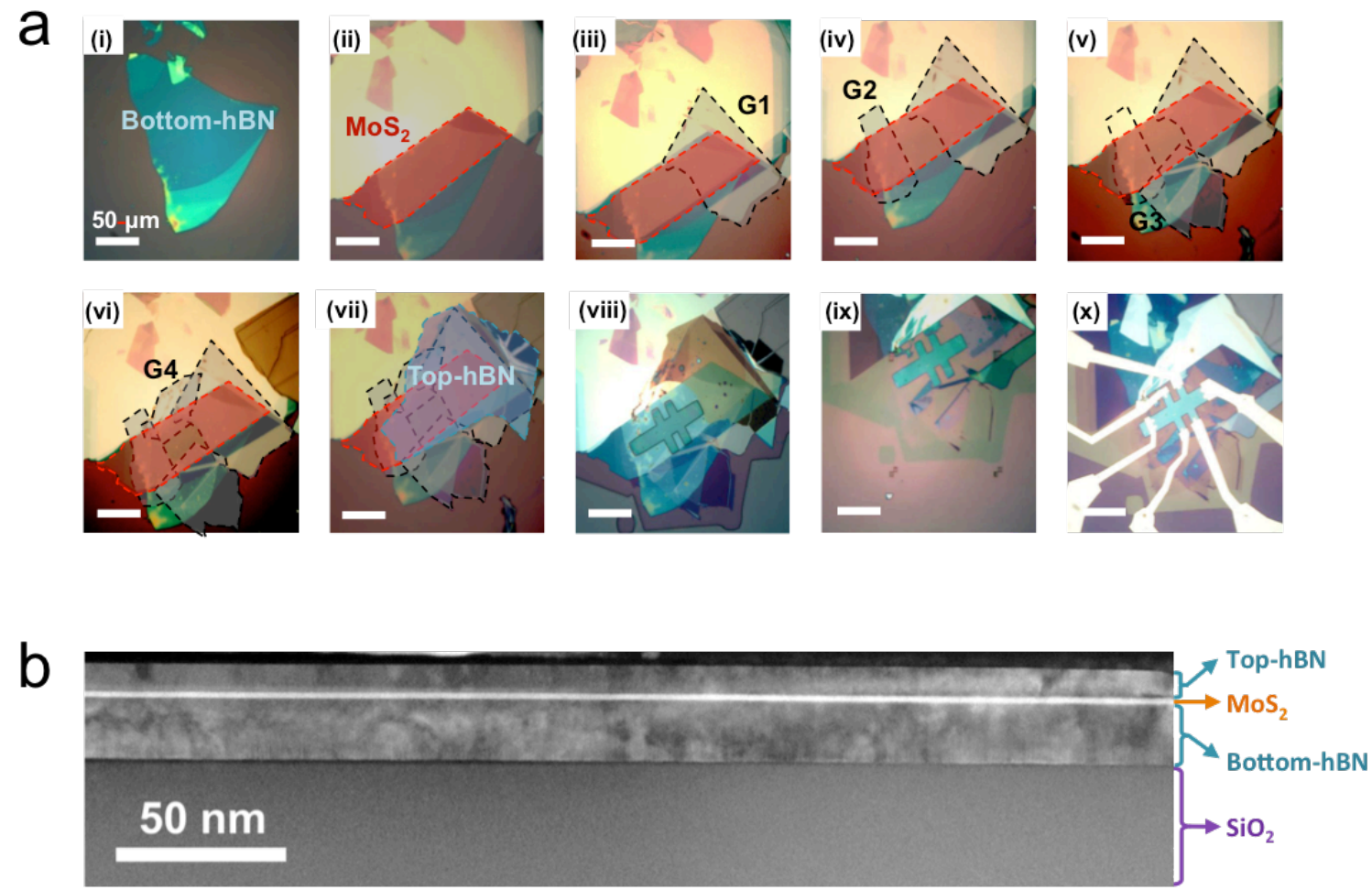

Supplementary Fig. 1 | Device fabrication process and ultra-clean heterointerfaces. a, Device fabrication process: (i) Bottom-hBN flake exfoliated onto a $\mathrm{SiO}_{2} / \mathrm{Si}$ substrate. (ii) $\mathrm{MoS}_{2}$ on $\mathrm{hBN}$. $\mathrm{MoS}_{2}$ was transferred onto bottom-hBN. (iii-vi) Four graphene flakes on hBN/MoS stack. Four graphene flakes were sequentially transferred onto the stack around the perimeter of the $\mathrm{MoS}_{2}$ channel. (vii) Top-hBN on graphene/MoS$/ \mathrm{hBN}$ stack. Top-hBN was transferred on top of the stack for encapsulation. (viii) Patterning of PMMA into Hall bar geometry. PMMA layer in the stack was patterned by e-beam lithography into a Hall bar configuration. (ix) Dry etching of the stack. The stack is etched using PMMA as an etch mask. (x) Formation of metal leads. Metal leads are fabricated by e-beam lithography and deposition of metals. Scale bar: 50 $\mu \mathrm{m}$. b, Cross-section STEM image of the stack (from top to bottom: hBN $(8 \mathrm{~nm}), \mathrm{MoS}_{2}(3 \mathrm{~L})$, hBN (19 nm)). Scale bar: $50 \mathrm{~nm}$. 


\section{S2. Identification of $\mathrm{MoS}_{2}$ flakes on PDMS}

We used Raman spectroscopy and photoluminescence (PL) measurements (in Via, Renishaw, $532 \mathrm{~nm}$ laser) to identify the number of layers of $\mathrm{MoS}_{2}$ flakes on $\mathrm{PDMS}^{3,4}$. Figure S2a shows the PL spectra of a CVD-grown monolayer $\mathrm{MoS}_{2}$. Bright-field optical images of few-layer $\mathrm{MoS}_{2}$ exfoliated on PDMS are shown in Fig. S2b. The number of layers of these flakes was confirmed by the Raman spectra in Fig. S2c. We confirmed that $\mathrm{MoS}_{2}$ flakes on PDMS have the correlation between Raman peak position difference of $\mathrm{E}^{1}{ }_{2 \mathrm{~g}}$ and $\mathrm{A}_{1 \mathrm{~g}}$ modes and number of layers, which is consistent to our previous report ${ }^{4}$. 

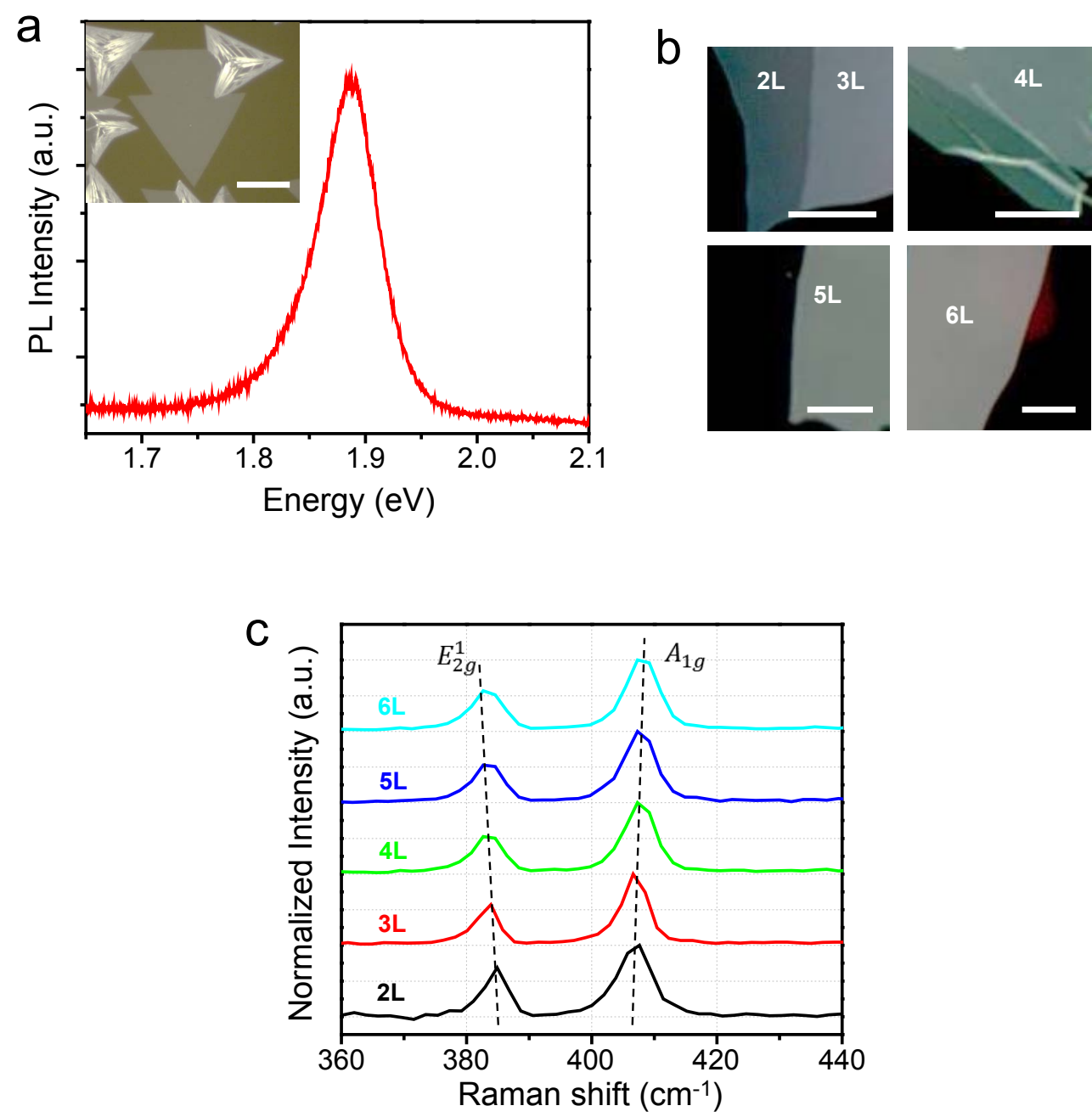

Supplementary Fig. 2 | Photoluminescence and Raman spectra of $\mathrm{MoS}_{2}$ on PDMS a, PL spectra of CVD 1L MoS 2 on PDMS. The inset shows bright field optical image of CVD 1L $\mathrm{MoS}_{2}$ on PDMS. b, Bright-field optical images of $2 \mathrm{~L}$ to $6 \mathrm{~L} \mathrm{MoS}_{2}$ flakes exfoliated on PDMS. Scale bar: $10 \mu \mathrm{m}$. c, Raman spectra of $2 \mathrm{~L}$ to $6 \mathrm{~L} \mathrm{MoS}_{2}$ on PDMS. As guided by the dashed lines, Raman peak position difference between $\mathrm{E}_{2 \mathrm{~g}}^{1}$ and $\mathrm{A}_{1 \mathrm{~g}}$ modes increases with $\mathrm{MoS}_{2}$ thickness. 


\section{S3. Contact resistance and Schottky barrier of graphene-MoS $\mathrm{M}_{2}$ contacts}

We estimate the contact resistance as $R_{c}=\frac{1}{2}\left(R_{2 P}-R_{4 P} \frac{L}{l}\right)$, where $R_{2 P}$ is two-probe resistance, $R_{4 P}$ is the four-probe resistance of $\mathrm{MoS}_{2}, L$ is the two-probe length and $l$ is fourprobe length. The calculated contact resistance as a function of temperature and back gate voltage is shown in Fig. S3. Due to increase of the $\mathrm{MoS}_{2}$ band gap with decreasing thickness from few-layers to monolayer ${ }^{3}$, it is more difficult to form Ohmic contact, in other words lower Schottky barrier, for thinner $\mathrm{MoS}_{2}{ }^{1}$. In this regard, graphene has been proved to be one of potential candidates as electrode for $\mathrm{MoS}_{2}{ }^{5,6}$. A large gate-tunability of Fermi energy of graphene enables us to move graphene's Fermi level close to conduction band of $\mathrm{MoS}_{2}$ by increasing back-gate voltage, resulting in reliable and stable Ohmic contact even for monolayer $\mathrm{MoS}_{2}$ as shown in Fig. S3a. At reasonably high charge carrier concentration, contact resistance from $\sim 0.7$ $\mathrm{k} \Omega \cdot \mu \mathrm{m}$ to $10 \mathrm{k} \Omega \cdot \mu \mathrm{m}$ can be reliably achieved across all samples at low temperature. 

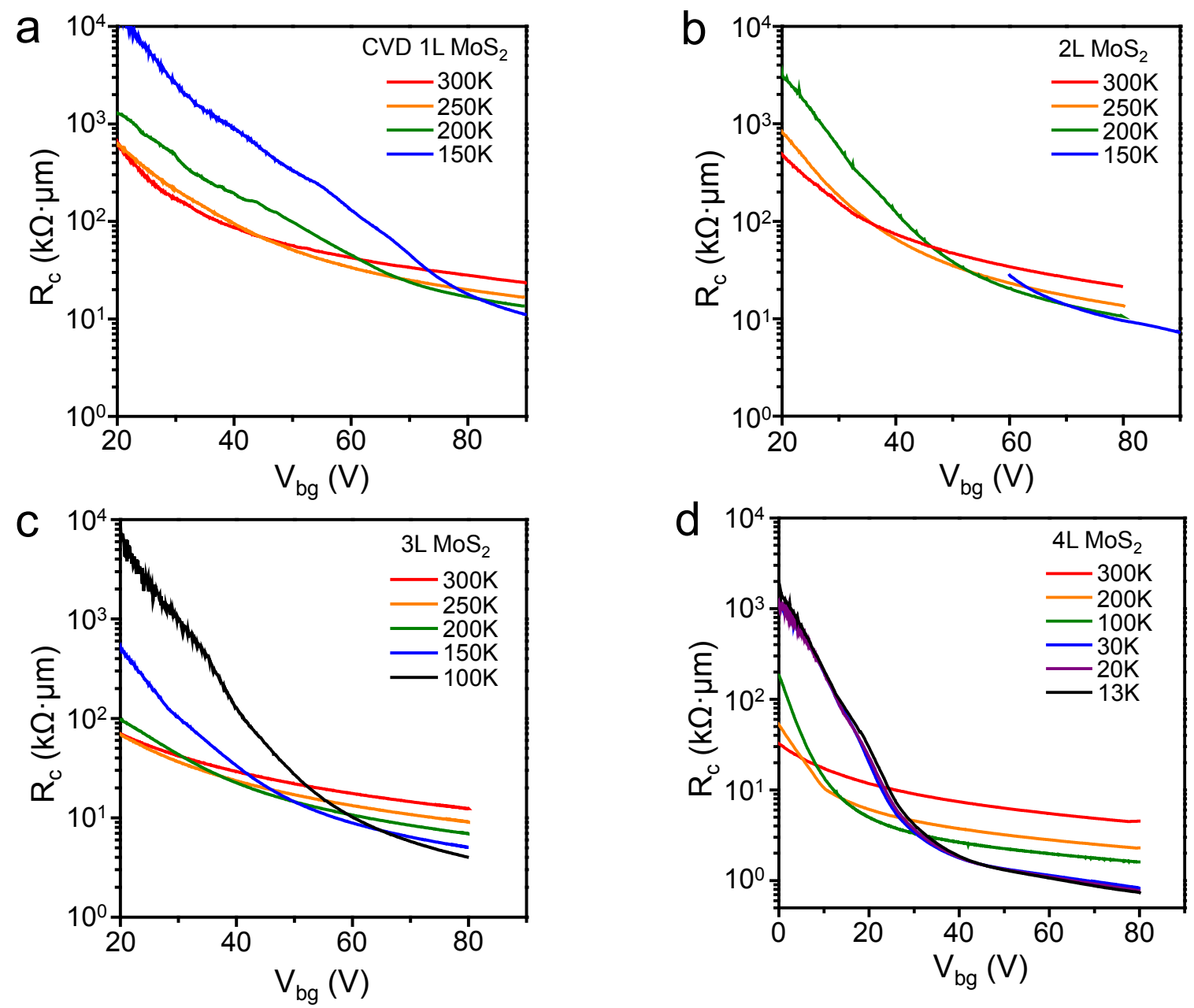

Supplementary Fig. 3 | Gate-tunable contact resistance of graphene-MoS $\mathbf{2}_{\mathbf{2}}$ contact. Contact resistance as a function of back-gate voltage $\left(\mathrm{V}_{\mathrm{bg}}\right)$ and temperature for $1 \mathrm{~L}(\mathrm{CVD}), 2 \mathrm{~L}, 3 \mathrm{~L}$ and $4 \mathrm{~L}$.

Furthermore, we calculated the Schottky barrier height of graphene- $\mathrm{MoS}_{2}$ contact using the 2D thermionic emission relation ${ }^{5}$,

$$
I_{d}=A T^{3 / 2} \exp \left(\frac{-q \phi_{B}}{k_{B} T}\right)\left[\exp \left(\frac{q V_{\mathrm{ds}}}{\eta k_{B} T}\right)-1\right]
$$

where $I_{d}, A, T, k_{B}, q, \phi_{B}$, and $V_{\mathrm{ds}}$ are drain current, the effective Richardson constant, temperature, the Boltzmann constant, electronic charge, the Schottky barrier height, and sourcedrain bias $(50 \mathrm{mV})$, respectively. Here, $\eta$ is an ideality factor, which is related with tunneling 
effect contribution under high charge carrier concentration and at low temperature. To estimate the Schottky barrier height of graphene- $\mathrm{MoS}_{2}$ with different back gate voltage $\left(\mathrm{V}_{\mathrm{bg}}\right)$, we employed the Arrhenius plot, $\ln \left(I_{d} / T^{3 / 2}\right)$ as a function of $1 / k_{B} T$ as shown in Fig. S4. Because the slope of Arrhenius plot is the $-q \phi_{B}+q V_{\mathrm{ds}} / \eta$, we can extract the Schottky barrier heights for different $\mathrm{MoS}_{2}$ thickness. Here, we assume the ideality factor as $2<\eta<20$, and we check the availability of ideality factor from the Ohmic behavior in 2 probe output curve at low temperature. Fig. S5 exhibits the calculated Schottky barrier heights in hBN-encapsulated $\mathrm{MoS}_{2}$ devices with different $\mathrm{MoS}_{2}$ thickness. Large modulation of graphene's Fermi energy allows for the high tunability of the Schottky barrier height in graphene-MoS $\mathrm{S}_{2}$ contact, resulting in the small Schottky barrier height with relatively high $\mathrm{V}_{\mathrm{bg}}$. The Schottky barrier height becomes close to zero at $\mathrm{V}_{\mathrm{bg}}$ of $\sim 80 \mathrm{~V}$ even for monolayer $\mathrm{MoS}_{2}$, which enable us to form the Ohmic contact at very low temperature. 

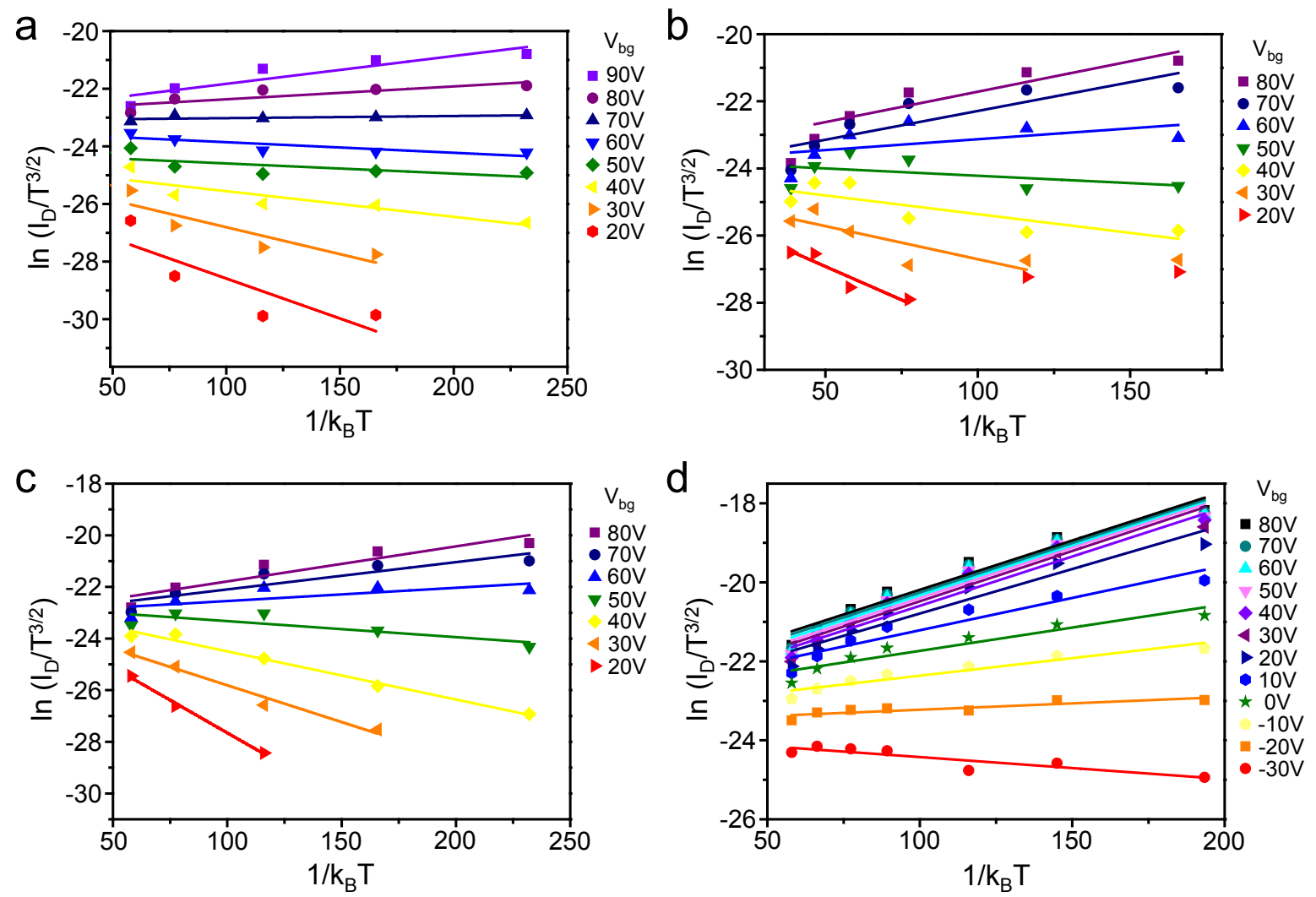

Supplementary Fig. 4 | Arrhenius plots of hBN-encapsulated $\mathrm{MoS}_{2}$ devices for calculations of the Schottky barrier heights. a, 1L (CVD) b, 2L c, 3L d, 4L MoS 2. 


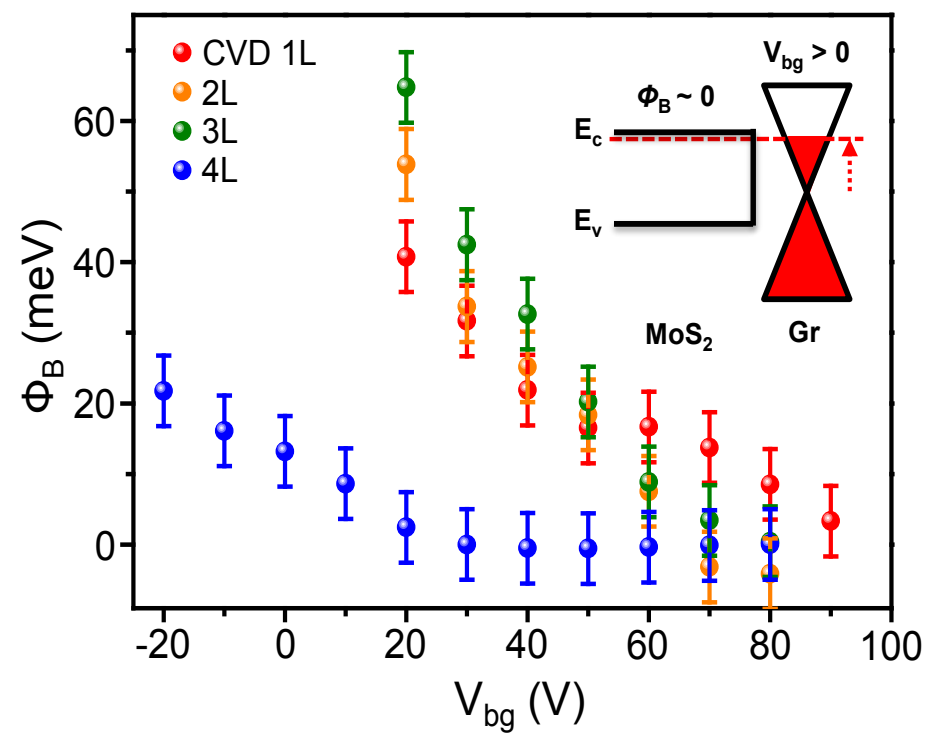

Supplementary Fig. 5 | Gate-voltage-tunability of the Schottky barrier heights in graphene$\mathbf{M o S}_{2}$ contacts. At higher back gate voltage, the Schottky barrier height can be reduced by modulation of graphene's Fermi level. The absence of Schottky barrier height enable us to form the Ohmic contact even at very low temperature. 


\section{S4. Hall measurements and Hall mobility calculations}

In an hBN-encapsulated $\mathrm{MoS}_{2}$ device, the Hall voltage and Hall mobility was measured with standard lock-in technique. In Fig. S6a, the Hall resistance $\left(R_{x y}\right)$ was plotted as a function of magnetic field $(B)$ at different $\mathrm{V}_{\mathrm{bg}}$. The total charge carrier concentration of $\operatorname{MoS}_{2}(n)$ is obtained

from $n_{\text {Hall }}=\frac{1}{e}\left(\frac{d B}{d R_{x y}}\right)$, where $e$ and $\left(\frac{d B}{d R_{x y}}\right)$ are electron charge and magnetic-field-dependence of Hall resistance. As shown in Fig. S6a, $R_{x y}$ is linearly dependent on the magnetic field at $10 \mathrm{~K}$ and the calculated total charge carrier concentration has a linear relationship with applied back gate voltage $\left(\mathrm{V}_{\mathrm{bg}}\right)$ as shown in Fig. S6b. These results confirm the validity of our Hall measurement at low temperature. With total charge carrier concentration, we estimate the Hall mobility from $\mu_{\text {Hall }}=\sigma / n e$, where $\mu_{\text {Hall }}$ is Hall mobility, $\sigma$ is conductivity, $n$ is total charge carrier concentration of $\mathrm{MoS}_{2}$ and $e$ is the electron charge. 

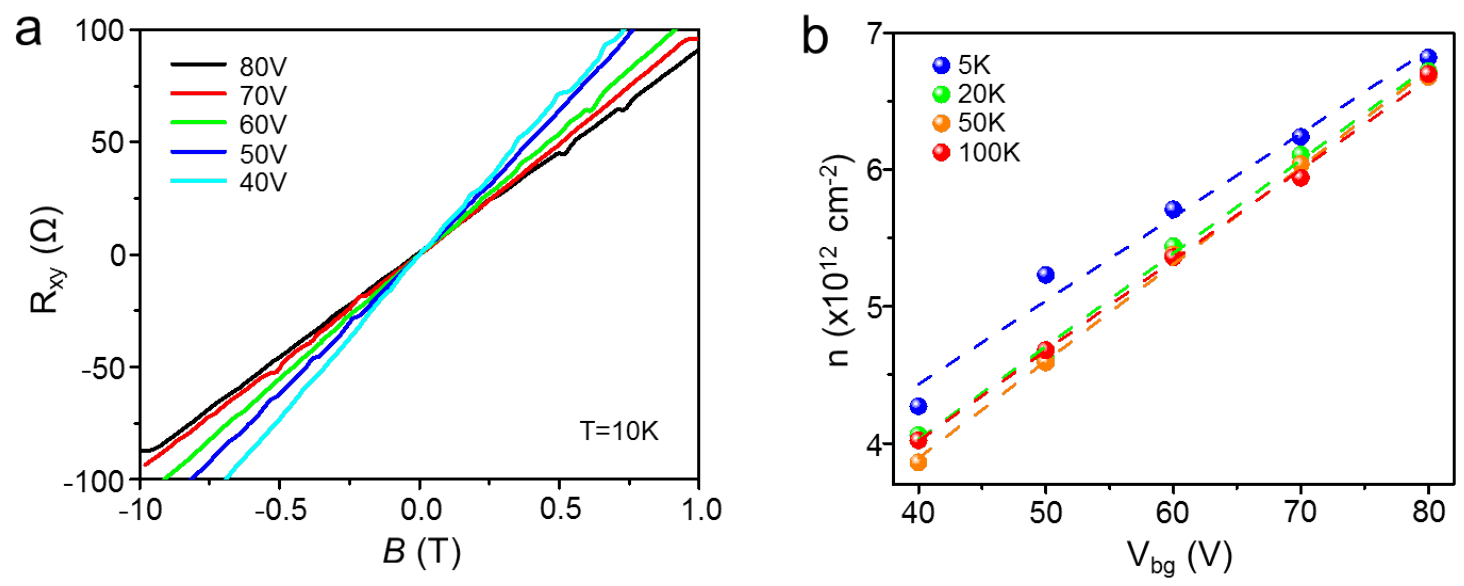

Supplementary Fig. 6 | Hall measurement and charge carrier concentration of $\mathrm{MoS}_{2}$. a, Hall resistance $\left(R_{x y}\right)$ at $10 \mathrm{~K}$ as a function of magnetic field $(B)$ at different back gate voltage $\left(\mathrm{V}_{\mathrm{bg}}\right)$ for $6 \mathrm{~L} \mathrm{MoS}_{2}$. b, Charge carrier concentration of $\mathrm{MoS}_{2}$ (n) calculated from Hall measurement at various temperatures as a function of $\mathrm{V}_{\mathrm{bg}}$ for $6 \mathrm{~L} \mathrm{MoS}_{2}$. The charge carrier concentration linearly increases as a function of back gate voltage.

\begin{tabular}{cccc}
\hline Layer Number & $\boldsymbol{\mu}_{\mathbf{i m p}}\left(\mathbf{c m}^{2} / \mathbf{V s}\right)$ & Carrier Density $\left(\mathbf{c m}^{-2}\right)$ & $\boldsymbol{V}_{\mathbf{p h}}$ \\
\hline 1L (CVD) & 1,020 & $1.1 \times 10^{13}$ & 1.9 \\
\hline 2L & 3,350 & $6.9 \times 10^{12}$ & 2.5 \\
\hline 3L & 1,530 & $9.3 \times 10^{12}$ & 2.0 \\
\hline 4L & 7,300 & $6.9 \times 10^{12}$ & 2.2 \\
\hline $6 \mathrm{~L}$ & 34,000 & $6.8 \times 10^{12}$ & 2.3 \\
\hline 1L (Ref.S7) & 264 & $1.9 \times 10^{13}$ & 1.7 \\
\hline 2L (Ref.S7) & 394 & $1.3 \times 10^{13}$ & 1.1 \\
\hline 4L (Ref.S8) & 314 & $1.2 \times 10^{13}$ & 1.5 \\
\hline
\end{tabular}

Supplementary Table 1 | Comparison and fitting parameters for hBN-encapsulated $\mathrm{MoS}_{2}$ $\operatorname{devices}^{7,8}$. 


\section{S5. Optical phonon-limited mobility in $\mathrm{MoS}_{2}$}

To attain $\gamma$ values of $\mathrm{hBN}$-encapsulated $\mathrm{MoS}_{2}$ devices, we fitted the Hall mobility with temperature as shown in Fig. S7. We assumed Coulomb impurity scattering and phonon scattering as the dominant scattering mechanisms in our work. By applying Matthiessen's rule, total mobility can be expressed with $\frac{1}{\mu(T)}=\frac{1}{\mu_{i m p}}+\frac{1}{\mu_{p h}(T)}$, where $\mu_{i m p}$ is the Coulomb impurity limited mobility at low temperature and $\mu_{p h}$ is the one affected by optical phonon of $\mathrm{MoS}_{2}$ dominant above $100 \mathrm{~K}^{9}$. From the Matthiessen's rule, optical phonon-limited mobility (Fig. S7) was extracted by subtracting impurity-limited mobility from the measured Hall mobility and could be well fitted by a power law, $\mu_{p h} \sim T^{-\gamma}$, indicating that optical phonon scattering is dominant above $100 \mathrm{~K}$. We obtained the relatively large $\gamma$ values of $1.9-2.5$ among our samples, which shows much stronger temperature-dependence of our hBN-encapsulated $\mathrm{MoS}_{2}$ devices compared to previously reported values of $0.7-1.7$ in $\mathrm{SiO}_{2}$-supported $\mathrm{MoS}_{2}$ devices. 


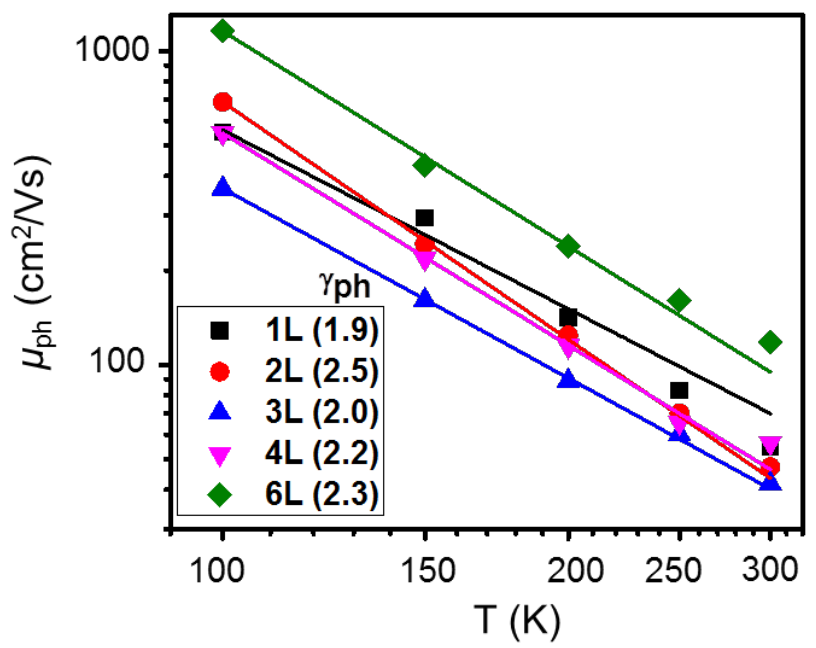

Supplementary Fig. 7 | Optical phonon-limited mobility of $\mathbf{M o S}_{2}$. The phonon-limited mobilities $\left(\mu_{\mathrm{ph}}\right)$ of $\mathrm{hBN}$-encapsulated $\mathrm{MoS}_{2}$ devices with different $\mathrm{MoS}_{2}$ thickness are plotted in the temperature range of $100-300 \mathrm{~K}$. By fitting with above $100 \mathrm{~K}$, the values of $\gamma$ were obtained as shown in the inset of Fig. 3a. 


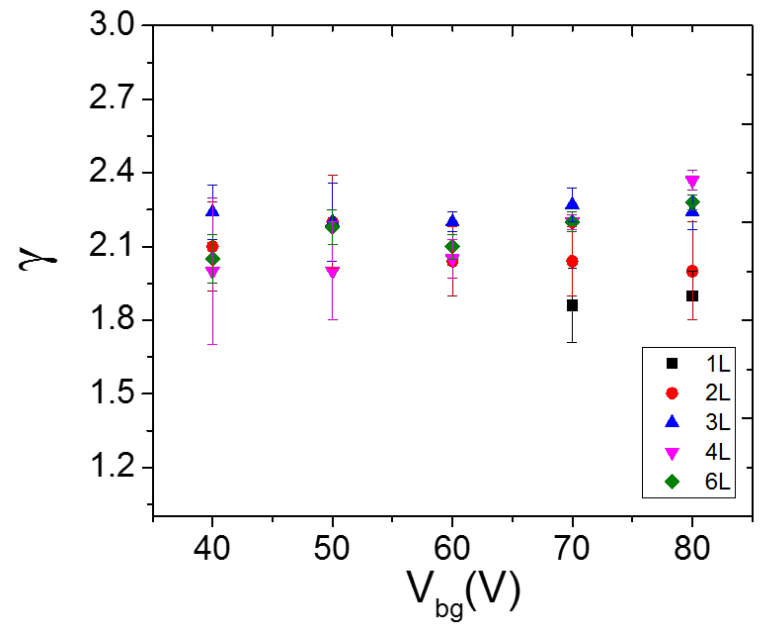

Supplementary Fig. $8 \mid \gamma$ as function of gate voltages. Estimated values of $\gamma$ as function of gate voltage (charge carrier density) from different $\mathrm{MoS}_{2}$ thickness are within the experimental uncertainty. It suggests that electron-optical phonon coupling in $\mathrm{MoS}_{2}$ is not dynamically screened by gate voltage. Notice this plot is calculated based on field-effect mobilites. 


\section{S6. Quantum scattering time analysis in $\mathbf{M o S}_{2}$}

We calculate the quantum scattering time in $\mathrm{MoS}_{2}$ from Shubnikov-de Haas (SdH) oscillations. Comparison of the ratio of the transport scattering time to quantum scattering time by number of layer of $\mathrm{MoS}_{2}$ will provide the deep understanding about scattering sources in $\mathrm{MoS}_{2}$. First, transport scattering time $\tau_{t}=m^{*} \mu / e$ are obtained from Hall mobility of $\mathrm{MoS}_{2}$. And, to obtain the quantum scattering time, we used the Ando formula, $\frac{\delta \rho_{x x}}{\rho_{0}}=4 \gamma_{t h} \exp \left(-\frac{\pi}{\omega_{c} \tau_{q}}\right)$, where $\gamma_{t h}=\frac{2 \pi^{2} k_{B} T / \hbar \omega_{c}}{\sinh \left(2 \pi^{2} k_{B} T / \hbar \omega_{c}\right)}$ is Dingle term, $\omega_{c}$ is the cyclotron frequency and $\tau_{q}$ is the quantum scattering time. We also calculate the quantum scattering time from quantum mobility, which are estimated from the onset of $\mathrm{SdH}$ oscillation magnetic field. We note that band structure calculations of $\mathrm{MoS}_{2}$ predict that there are two bands (at the $\mathrm{K}$ and $\Lambda$ points) near the Fermi level, and that for $1 \mathrm{~L}$ to $3 \mathrm{~L}$, the $\mathrm{K}$ band $\left(m^{*} \sim 0.5 m_{0}\right)$ is lowest in energy, meanwhile the $\Lambda$ band $\left(m^{*} \sim\right.$ $\left.0.6 m_{0}\right)$ is lowest for $>4 \mathrm{~L}^{10}$. Figure S9d exhibits the $\tau_{t} / \tau_{q}$ ratio increase as increase of number of layer, and it is due to the long-range scattering origins $\left(\tau_{t} / \tau_{q}>1\right)$ such as charged impurities and adsorbents become the dominant source of scattering in few-layer $\mathrm{MoS}_{2}$ which lead dominant small angle scattering that destroy cyclotron orbit motions, while the short-range scattering origins $\left(\tau_{t} / \tau_{q} \sim 1\right)$ such as vacancies, ripple and cracks are dominant at the monolayer $\mathrm{MoS}_{2}$ by the nature of 2D materials. 
a

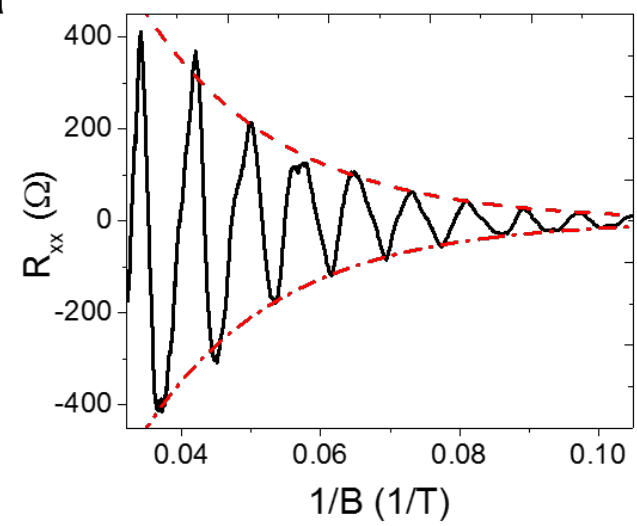

C

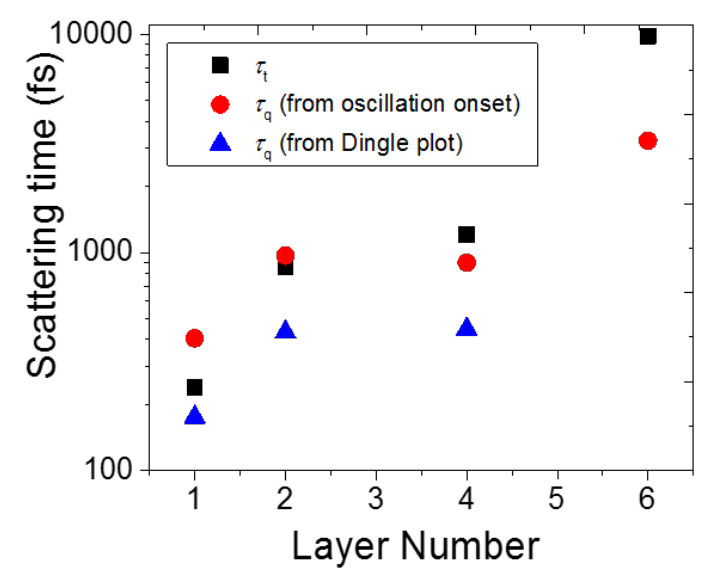

$\mathrm{b}$

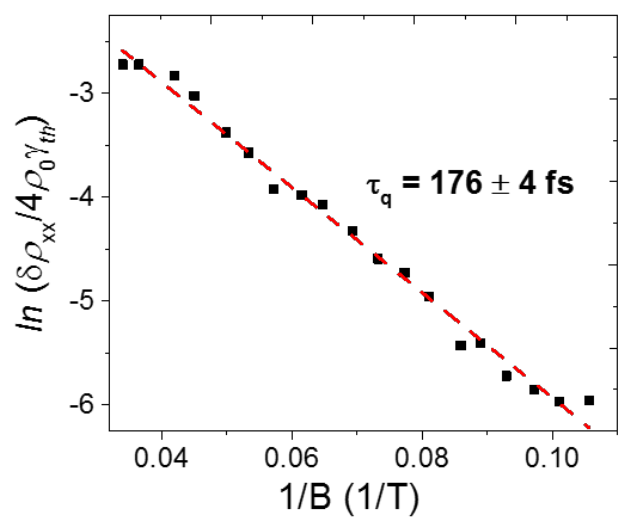

d

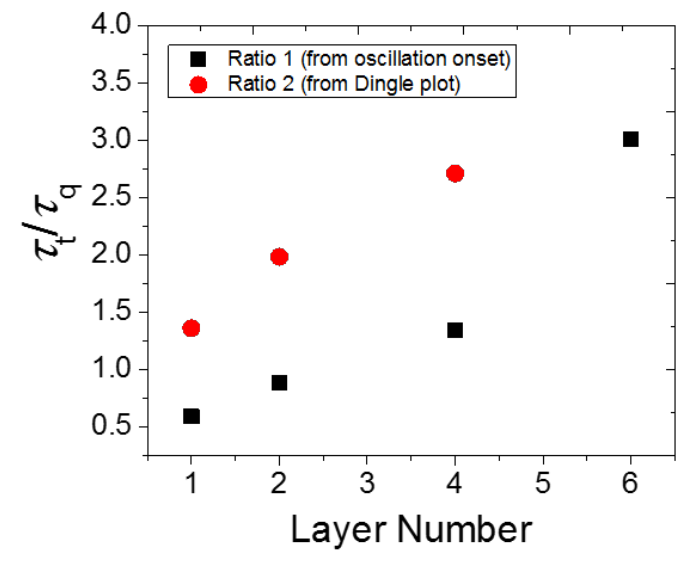

Supplementary Fig. 9 | Quantum scattering time of $\mathrm{MoS}_{2}$. a, $\mathrm{R}_{\mathrm{xx}}$ as function of inverse magnetic field of monolayer $\mathrm{MoS}_{2}$ (solid line) as shown in Fig. 4a and dashed lines correspond to the Ando formula. b, Quantum scattering time $\left(\tau_{q}=176 \pm 4 \mathrm{fs}\right)$ of monolayer $\mathrm{MoS}_{2}$ are extracted from the Dingle plot. c, Estimated scattering times as function of number of layer of $\mathrm{MoS}_{2}$. d, Ratio of the transport and quantum scattering time (red: from Dingle plot, black: from $\mathrm{SdH}$ oscillation onset) as a function of number of layer of $\mathrm{MoS}_{2}$. 


\section{S7. Periodicity analysis of SdH oscillations in monolayer $\mathrm{MoS}_{2}$}

We estimate the charge carrier concentration $\left(n_{S d H}\right)$ from the $\mathrm{SdH}$ oscillation of monolayer $\mathrm{MoS}_{2}$ with different gate voltage as shown in Fig. S10a. We analysis the $\mathrm{SdH}$ oscillations peaks as function of inverse magnetic field with different gate voltage and we can clearly see the periodicity change as shown in Fig. S10a and S10b. When we compare the total charge carrier concentration $\left(n_{t}\right)$ from the Hall measurements with $n_{S d H}$, we have to consider the degeneracy of monolayer $\mathrm{MoS}_{2}$. First, when we assume the spin and valley degeneracy $(g=4)$ in monolayer $\mathrm{MoS}_{2}, n_{S d H}$ is larger than $n_{t}$ as shown in Fig. S10c. So degeneracy in monolayer MoS 2 should be smaller than 4 , it may be attribute to the strong spin-orbit coupling induced lift of the spin degeneracy $(\Delta E=1 \sim 10 \mathrm{meV})$ at conduction band of monolayer $\mathrm{MoS}_{2}$. So we need further study about the lift of spin or valley degeneracy of $\mathrm{MoS}_{2}$ by strong spin-orbit coupling and Zeeman effect under high magnetic field. 

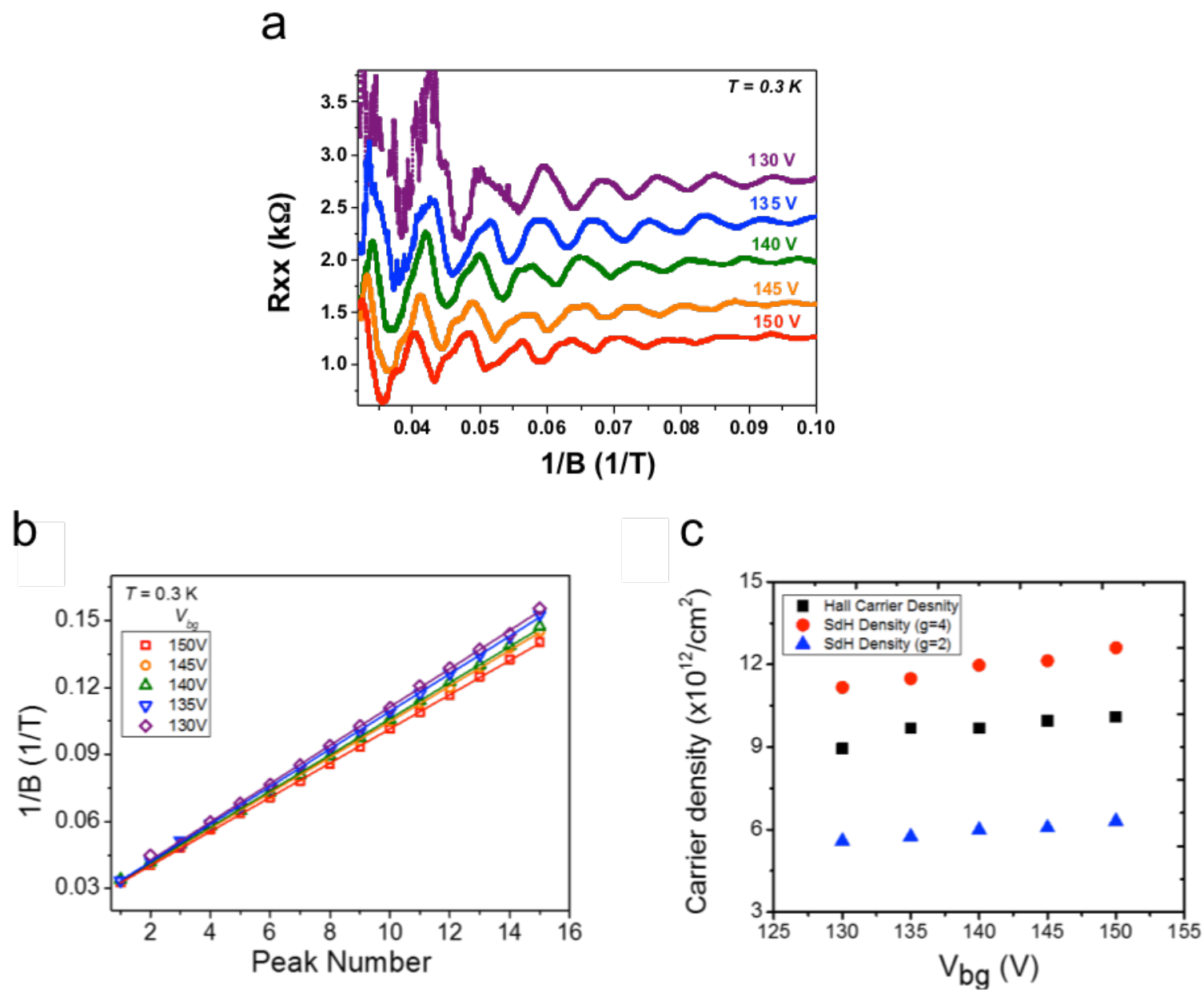

Supplementary Fig. 10 | Periodicity analysis of monolayer $\mathrm{MoS}_{2} \cdot \mathbf{a}, \mathrm{R}_{\mathrm{xx}}$ of monolayer $\mathrm{MoS}_{2}$ as a function of inverse magnetic field with varying gate voltage. $\mathbf{b}, \mathrm{SdH}$ oscillations peaks as function of inverse magnetic field with different gate voltage. c, Estimated charge carrier concentration from SdH oscillation with different degeneracy (red: $g=4$, blue: $g=2$ ) and total charge carrier concentration from Hall measurements (black) of monolayer $\mathrm{MoS}_{2}$. 
S6. Modeling $\mathrm{MoS}_{2}$ electron mobility limited by interfacial Coulomb impurities

\section{A. Electrostatics}

The problem consists of a thin layer of semiconductor of thickness $t_{s}, \mathrm{MoS}_{2}$ in this case, sandwiched between two dielectrics layers. Let $z$ denotes the direction normal to these layers, and $m_{z}$ be the quantization mass of $\mathrm{MoS}_{2}$. We uses a triangular well approximation as an initial estimate to the electrostatics of $\mathrm{MoS}_{2}$ in the confinement direction, where $F_{s}$ is its electric field, where the eigen-solutions are known ${ }^{8}$. The eigen-energies $E_{j}$ must satisfy,

$$
\operatorname{Ai}\left(z_{0}\right) \operatorname{Bi}\left(z_{1}\right)-\operatorname{Ai}\left(z_{1}\right) \operatorname{Bi}\left(z_{0}\right)=0
$$

where $\mathrm{Ai}$ and $\mathrm{Bi}$ are the Airy functions, and

$$
z_{0}=-\left(\frac{2 m_{z}}{\hbar^{2} e^{2} F_{s}^{2}}\right)^{1 / 3} E_{j}, z_{1}=\left(\frac{2 m_{z} e F_{s}}{\hbar^{2}}\right)^{1 / 3}\left(t_{s}-\frac{E_{j}}{e F_{s}}\right)
$$

The eigen-functions are then given by,

$$
\psi_{j}(z)=a A i\left[\left(\frac{\pi}{v}\right)^{2 / 3}\left(\frac{v z}{t_{s}}-\gamma\right)\right]+b B i\left[\left(\frac{\pi}{v}\right)^{2 / 3}\left(\frac{v z}{t_{s}}-\gamma\right)\right]
$$

where $v=e F_{s} t_{s} / \eta, \eta=\hbar^{2} \pi^{2} / 2 m_{z} t_{s}^{2}$ and $\gamma=E_{j} / \eta$. Within this triangular model approximation, the electric fields in the different regions are related via $\epsilon_{o x 1} F_{o x 1}=\epsilon_{s} F_{s}=\epsilon_{o x 2} F_{o x 2}$. The carrier densities can be computed from,

$$
\begin{aligned}
n & =\sum_{i, v} \frac{g_{v} m_{d}^{v}}{\pi \hbar^{2}} \int_{E_{i, v}}^{\infty} \frac{1}{\exp \left(\frac{E-E_{f}}{k T}\right)+1} d E\left|\psi_{i, v}(z)\right|^{2} \\
& =\frac{k T}{\pi \hbar^{2}} \sum_{i, v} g_{v} m_{d}^{v} \ln \left[\exp \left(\frac{E_{f}-E_{i, v}}{k T}\right)+1\right]\left|\psi_{i, v}(z)\right|^{2}
\end{aligned}
$$

where $i$ and $v$ denotes the subbands and valleys. $g_{v}$ is the valley degeneracy. $m_{d}$ is the density of states mass. The Fermi energy $E_{f}$ is determined from Eq. 3, by imposing that $n=C_{o x} V_{g}$.

The solutions to the triangular well approximation provides an initial guess to the electrostatics. With this, we solved the multilayers $\mathrm{MoS}_{2}$ electrostatics by solving the Poisson and Schrödinger equation self-consistently within the effective mass framework. In this work, we include both the $K$ and $\Lambda$ valleys, with band edge energies that are close to one another in multilayers $\mathrm{MoS}_{2}$. With reference to calculations obtained from density 
functional theory ${ }^{11-14}$, we extract the in-plane and out-of-plane masses: $m_{d, K}=0.5 m_{0}$, $m_{d, \Lambda}=0.6 m_{0}, m_{z, K}=1.5 m_{0}, m_{z, K}=1.0 m_{0}$. Due to their different $m_{z}$, the two valleys have different band edge energy offset depending on the $\mathrm{MoS}_{2}$ thickness, and is taken to be zero when $t_{s}=4 \mathrm{~nm}$. Their valley degeneracies are $g_{v, K}=2$ and $g_{v, \Lambda}=6$.

\section{B. Screened Interfacial Coulomb potential}

To calculate the scattering rate due to Coulomb centers, we must first find the scattering potential induced by a point charge. This Coulomb potential is governed by the following Poisson equation;

$$
\nabla \epsilon(\vec{r}, z) \nabla \phi\left(\vec{r}, z, z_{0}\right)=-e \delta\left(z-z_{0}\right)-e \rho_{\text {ind }}
$$

where $\vec{r}$ is the $2 D$ position vector describing the plane perpendicular to the gate confinement direction. The presence of the external point charge resulted in a Coulomb potential $\phi\left(\vec{r}, z, z_{0}\right)$ which also induced charge $\rho_{\text {ind }}$. Next, we need to obtain an expression for $\rho_{\text {ind }}$.

Here, we employed a perturbative approach by Stern ${ }^{9}$ commonly used in the context of semiconductor devices. The presence of the perturbation potential $\phi(z)$ results in correction of the eigen-energies $\delta E_{i, v}=-e \int \phi(z)\left|\psi_{i, v}(z)\right|^{2} d z$. The charge induced $\rho_{\text {ind }}$ is the change in the amount of charge due to the change in eigen-energies $\delta E_{i, v}$;

$$
\begin{aligned}
\rho_{\text {ind }}(z) & =-e \sum_{i, v} \frac{d n_{i, v}}{d E_{i, v}} \delta E_{i, v} \\
& =-\sum_{i, v} \frac{g_{v} m_{d}^{v} e^{2}}{\pi \hbar^{2}} \frac{1}{1+\exp \left(\frac{E_{i, v}-E_{f}}{k T}\right)}\left|\psi_{i, v}(z)\right|^{2} \int \phi\left(z^{\prime}\right)\left|\psi_{i, v}\left(z^{\prime}\right)\right|^{2} d z^{\prime} \\
& \equiv-\sum_{i, v} S_{i, v}\left|\psi_{i, v}(z)\right|^{2} \int \phi\left(z^{\prime}\right)\left|\psi_{i, v}\left(z^{\prime}\right)\right|^{2} d z^{\prime}
\end{aligned}
$$

Hence, Eq.4 can be approximated by the following;

$$
\nabla \epsilon(\vec{r}, z) \nabla \phi\left(\vec{r}, z, z_{0}\right)=-e \delta\left(z-z_{0}\right)+e \sum_{i, v} S_{i, v}\left|\psi_{i, v}(z)\right|^{2} \int \phi\left(\vec{r}, z^{\prime}\right)\left|\psi_{i, v}\left(z^{\prime}\right)\right|^{2} d z^{\prime}
$$

This is the key result by Stern ${ }^{9}$.

It renders the problem easier to express the in-plane part of thr potential $\phi\left(\vec{r}, z, z_{0}\right)$ in its $2 D$ Fourier representation,

$$
\phi\left(\vec{r}, z, z_{0}\right)=\sum_{\vec{q}} \tilde{\phi}_{\vec{q}} \exp (i \vec{q} \cdot \vec{r}) \phi_{0}\left(z, z_{0}\right)
$$


Thus we have the Poisson equation in the semiconductor region;

$$
\epsilon_{s c} \nabla^{2} \phi_{0}\left(z, z_{0}\right)-\epsilon_{s c} q^{2} \phi_{0}\left(z, z_{0}\right)=-e \delta\left(z-z_{0}\right)+\underbrace{\sum_{i, v} S_{i, v}\left|\psi_{i, v}(z)\right|^{2} \int \phi_{0}\left(z^{\prime}, z_{0}\right)\left|\psi_{i, v}\left(z^{\prime}\right)\right|^{2} d z^{\prime}}_{K\left(z_{1}, z_{0}\right)}
$$

Here, $\epsilon_{s c}=7.4$ is the out-of-plane dielectric constant of $\mathrm{MoS}_{2}{ }^{10}$. Multiply by $1 / q \cdot \exp (-q \mid z-$ $\left.z_{1} \mid\right)$ with $0<z<t_{s}$ and integrating for $0<z_{1}<t_{s}$, we arrived at,

$$
\phi_{0}\left(z, z_{0}\right)=-\frac{e}{2 q \epsilon_{s c}} \int_{0}^{t_{s}} \exp \left(-q\left|z-z_{1}\right|\right) K\left(z_{1}, z_{0}\right) d z_{1}+\frac{e}{2 q \epsilon_{s c}} \int_{0}^{t_{s}} \exp \left(-q\left|z-z_{1}\right|\right) \delta\left(z_{1}-z_{0}\right) d z_{1}
$$

Note we assume the semiconductor is sandwiched by dielectrics, i.e. no metal gates. Otherwise, we have additional terms given by $A(q) \exp (-q z)+B(q) \exp (q z)$ to account for screening by the metals.

\section{Mobility}

With $\phi_{0}(\vec{q}, z, 0)$, one uses Fermi Golden rule to obtain the scattering probability;

$$
P\left(\vec{k}_{i}, \vec{k}_{j}\right)=N_{i m} \frac{2 \pi}{\hbar}\left|e \int_{0}^{\infty} \phi_{0}(\vec{q}, z, 0) \psi_{i}(z) \psi_{j}(z) d z\right|^{2} \delta\left(E\left(\vec{k}_{i}\right)-E\left(\vec{k}_{j}\right)\right)
$$

where $k_{i}=\sqrt{2 m_{d}^{i} / \hbar^{2}\left(E-E_{i}\right)}, \vec{q}=\overrightarrow{k_{j}}-\overrightarrow{k_{i}}$ and $\theta$ is the angle between the initial and final wave vector. $N_{i m}$ is the impurities concentration. To calculate the relaxation time, we write,

$$
\begin{aligned}
\frac{1}{\tau_{i}\left(\vec{k}_{i}\right)} & =\sum_{\vec{k}_{j}^{\prime}} P\left(\vec{k}_{i}, \vec{k}_{j}^{\prime}\right)\left[1-\frac{\tau_{j}\left(\vec{k}_{j}^{\prime}\right) k_{j}^{\prime} m_{c, j}}{\tau_{i}\left(\vec{k}_{i}\right) k_{i} m_{c, i}} \cos (\theta)\right] \delta\left(E\left(\vec{k}_{i}\right)-E\left(\vec{k}_{j}\right)\right) \\
\frac{1}{\tau_{i}\left(E_{i}\right)} & =\frac{m_{d, i}}{\pi \hbar^{3}} \sum_{j} \int_{0}^{\pi}\left|e \int_{0}^{\infty} \phi_{0}(\vec{q}, z, 0) \psi_{i}(z) \psi_{j}(z) d z\right|^{2} \delta\left(E_{i}-E_{j}\right)\left[1-\frac{\tau_{j}\left(E_{i}\right) k_{j}^{\prime} m_{c, j}}{\tau_{i}\left(E_{i}\right) k_{i} m_{c, i}} \cos (\theta)\right] d \theta \\
\Rightarrow \frac{1}{\tau_{i}(E)} & \equiv \sum_{j}\left\{A_{i, j}-B_{i, j} \frac{\tau_{j}(E)}{\tau_{i}(E)}\right\}
\end{aligned}
$$

where $m_{c}$ is the transport mass. The above can be re-expressed into an equivalent matrix form as follows;

$$
\left(\begin{array}{c}
1 \\
1 \\
1 \\
1 \\
\vdots
\end{array}\right)=\left(\begin{array}{ccccc}
\sum_{j} A_{1 j} & -B_{12} & -B_{13} & -B_{14} & \cdots \\
-B_{21} & \sum_{j} A_{2 j} & -B_{23} & -B_{24} & \cdots \\
-B_{31} & -B_{32} & \sum_{j} A_{3 j} & -B_{34} & \cdots \\
-B_{41} & -B_{42} & -B_{43} & \sum_{j} A_{4 j} & \cdots \\
\vdots & \vdots & \vdots & \vdots & \ddots
\end{array}\right)\left(\begin{array}{c}
\tau_{1}(E) \\
\tau_{2}(E) \\
\tau_{3}(E) \\
\tau_{4}(E) \\
\vdots
\end{array}\right)
$$

which can easily be solved by inverting the matrix above. The relaxation time can be similarly computed for the other valley. 
The mobility for each subband $j$ and valley $v$ then follows from,

$$
\mu_{j v}=-\frac{e}{m_{c, v}} \frac{\int\left(E-E_{f}\right) \tau_{j v}(E) \frac{\partial f\left(E, E_{f}\right)}{\partial E} d E}{\int f\left(E, E_{f}\right) d E}
$$

where $f\left(E, E_{f}\right)$ is the Fermi-Dirac distribution function. The total effective mobility is then

$$
\mu_{e f f}=\frac{\sum n_{j, v} \mu_{j, v}}{\sum n_{j, v}}
$$

where $n_{j, v}$ is the carrier densities in subband $j$ and valley $v$.

\section{Short-range scattering potential}

In the main manuscript, we discussed two tpyes of short-range scattering potential i.e. bulk and interfacial. The former could be due to atomic defects such as $\mathrm{S}$ vacancies in $\mathrm{MoS}_{2}$ itself, which is present in its natural crystal form. The latter, interfacial short-range scatterers, could be due to presence of chemical adsorbates that was introduced to the top interface due to subsequent processing steps after $\mathrm{MoS}_{2}$ transfer onto the $\mathrm{SiO}_{2}$ substrate. As explained in the main manuscript, we found the latter model to agree better to the experiments. We model the short-range scatterers at the top $\mathrm{MoS}_{2}$ interface (i.e. $z=0$ ) within the same framework describe above by simply making the replacement to the scattering potential in Eq. 9 i.e. $\phi_{0}(\vec{q}, z, 0) \rightarrow v_{s c} \delta(z)$ and $N_{s c}$ denotes the short-range scatterers concentration $\left(\mathrm{cm}^{-2}\right)$. On the other hand, the scattering potentials are distributed across the different layers of $\mathrm{MoS}_{2}$ when modeling of bulk short-range scatterings. In the case of $\mathrm{S}$ vacancies in $\mathrm{MoS}_{2}$, the total short-range scatterers concentration $\left(\mathrm{cm}^{-2}\right)$ increases with layer thickness and one expects a decrease in electron mobility with layer thickness contrary to what is observed in our experiments. We therefore focus only on interfacial short-range scattering potential in this work.

\section{E. Calculation results}

Here we show results of our calculation for both interfacial Coulomb impurities and short-range scatterers located at the top $\mathrm{MoS}_{2}$ interface. The long-range Coulomb limited moblity, $\mu_{L R}$, predicts a layer dependence weaker than the experiments of less than an order of magnitude within the range of carrier densities considered, while the short-range limited mobility, $\mu_{S R}$, predicts a much stronger thickness dependence larger than 4 orders of magnitude. In sum, their total contribution according to Matthiessen rule would allow for better quantitative agreement with the experiments. 

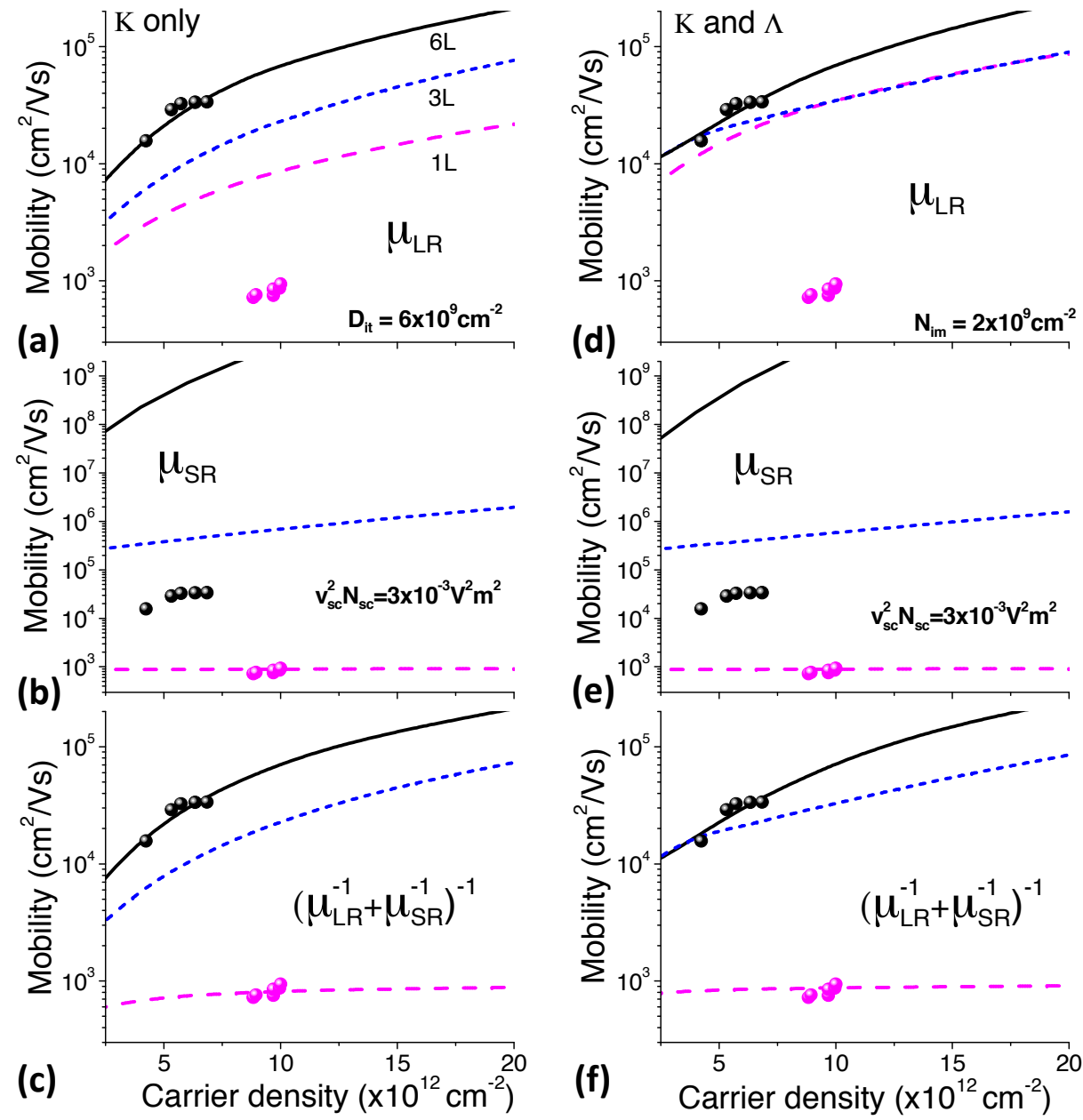

Fig. S8: Calculated electron mobility due to interfacial Coulomb impurities $\left(\mu_{L R}\right)$ and shortrange scatterers $\left(\mu_{S R}\right)$ located at the top $\mathrm{MoS}_{2}$ interface as function of gate bias and for different number of $\mathrm{MoS}_{2}$ layers. We consider two cases, one where only $K$ valleys are considered in (ac), and where both $K$ and $\Lambda$ valleys are included in (d-f). We also displayed the experimentally measured low temperature mobilities for $1 \mathrm{~L}$ and $6 \mathrm{~L}$ devices. We found that the calculated $\mu_{L R}$ predicts a layer dependence weaker than the experiments, while the calculated $\mu_{S R}$ predicts a much larger thickness dependence. In sum, their total contribution according to Matthiessen rule would allow for better quantitative agreement with the experiments. 


\section{References}

S1. Lee, GH. et al. Flexible and transparent $\mathrm{MoS}_{2}$ field-effect transistors on hexagonal boron nitride-graphene heterostructures. ACS Nano 7, 7931-7936 (2013).

S2. Wang, L. et al. One-Dimensional Electrical Contact to a Two-Dimensional Material. Science 342, 614-617 (2013).

S3. Mak, K., Lee, C., Hone, J., Shan, J. \& Heinz, T. Atomically Thin $\mathrm{MoS}_{2}$ : A New DirectGap Semiconductor. Phys. Rev. Lett. 105, 136805 (2010).

S4. Lee, C. et al. Anomalous lattice vibrations of single-and few-layer $\mathrm{MoS}_{2}$. ACS Nano 4, 2695-2700 (2010).

S5. $\quad \mathrm{Yu}$, L. et al. Graphene/MoS 2 Hybrid Technology for Large-Scale Two-Dimensional Electronics. Nano Lett. 14, 3055-3063 (2014).

S6. $\quad$ Roy, T. et al. Field-Effect Transistors Built from All Two-Dimensional Material Components. ACS Nano 8, 6256-6264 (2014).

S7. Baugher, B., Churchill, H., Yang, Y. \& Jarillo-Herrero, P. Intrinsic Electronic Transport Properties of High-Quality Monolayer and Bilayer $\mathrm{MoS}_{2}$. Nano Lett. 13, $4212-$ 4216 (2013).

S8. Neal, A., Liu, H., Gu, J. \& Ye, P. Magneto-transport in $\mathrm{MoS}_{2}$ : phase coherence, spinorbit scattering, and the hall factor. ACS Nano 7, 7077-1082 (2013).

S9. Ma, N. \& Jena, D. Charge Scattering and Mobility in Atomically Thin Semiconductors. Phys. Rev. X 4, 011043 (2014).

S10. Liu, G.-B., Shan, W.-Y., Yao, Y., Yao, W. \& Xiao, D. Three-band tight-binding model for monolayers of group-VIB transition metal dichalcogenides. Phys. Rev. B 88, 085433 (2013).

S11. Miller, David AB. "Quantum mechanics for scientists and engineers.” Cambridge University Press, Cambridge (2008)

S12. Stern, Frank, and W. E. Howard. "Properties of semiconductor surface inversion layers in the electric quantum limit." Phys. Rev. 163, 816 (1967)

S13. Molina-Sanchez, Alejandro, and Ludger Wirtz. "Phonons in single-layer and fewlayer $\mathrm{MoS}_{2}$ and $\mathrm{WS}_{2}$." Phys. Rev. B 84, 155413 (2011)

S14. Han, S. W., Hyuksang Kwon, et al. "Band-gap transition induced by interlayer van der Waals interaction in $\mathrm{MoS}_{2}$." Phys. Rev. B 84, 045409 (2011)

S15. Zhao, Weijie, Ricardo Mendes Ribeiro, et al. "Origin of Indirect Optical Transitions in Few-Layer $\mathrm{MoS}_{2}, \mathrm{WS}_{2}$, and WSe 2 ." Nano Lett. 13, 5627-5634 (2013)

S16. Wickramaratne, Darshana, Ferdows Zahid, and Roger K. Lake. "Electronic and thermoelectric properties of few-layer transition metal dichalcogenides." J. Chem. Phys. 140, 124710 (2014)

S17. Cheiwchanchamnangij, Tawinan, and Walter RL Lambrecht. "Quasiparticle band structure calculation of monolayer, bilayer, and bulk $\mathrm{MoS}_{2}$." Phys. Rev. B 85, 205302 (2012) 